\title{
belarus- analysen
}

\section{NACH DEM GIPFEL IN VILNIUS}

\section{EDITORIAL}

Belarus und die EU nach dem Gipfel in Vilnius

Astrid Sahm, Berlin

\section{ANALYSE}

Bewertungen des EU-Gipfels in Vilnius durch belarussische Experten

Multilateraler Ansatz der Östlichen Partnerschaft ohne Perspektiven

Denis Meljanzau, Vilnius

Drohende Demoralisierung der Zivilgesellschaft

Aljaksandr Adamjanz, Minsk

Die ukrainische Krise als Ausdruck des Scheiterns der EU-Außenpolitik 6

Andrej Kasakewitsch, Minsk

Zunehmende Integrationskonkurrenz zwischen Brüssel und Moskau als Chance für Belarus? $\quad 7$

Walerij Karbalewitsch, Minsk

Die EU-Politik des »more for more« ist nicht länger angemessen

Andrej Jahorau, Minsk

- UMFRAGE

Öffentliche Meinung zur Todesstrafe

- STATISTIK

Die Unterstützung internationaler Geber für Belarus 


\section{Belarus und die EU nach dem Gipfel in Vilnius}

Astrid Sahm, Berlin

$\mathrm{D}$ er EU-Gipfel in Vilnius stand ganz im Schatten der Entscheidung der ukrainischen Führung, vorerst auf eine Unterzeichnung des Assoziationsabkommens mit der EU zu verzichten. Bei unabhängigen belarussischen Experten sowie in der Lukaschenka-kritischen Öffentlichkeit dominiert offensichtlich die Enttäuschung über das Scheitern des Abkommens. Denn mit der Annäherung der Ukraine an die EU sollte ihrer Hoffnung nach auch den Bürgern in Belarus deutlich werden, dass die Europäische Union ihren osteuropäischen Nachbarstaaten eine reale Entwicklungsperspektive bietet. Freilich vermag sich auch die belarussische Führung über den offensichtlichen Erfolg Russlands, durch Druck eine weitergehende Anbindung der Ukraine und gleichfalls Armeniens - an die Europäische Union verhindert zu haben, nicht unbeschwert freuen. Denn sollte die Einbindung beider Staaten in die Zollunion bzw. die Eurasische Wirtschaftsunion gelingen, würde Belarus seine Sonderstellung als Russlands Integrationsköder für die anderen postsowjetischen Staaten verlieren. Damit muss die belarussische Führung um den Fortbestand der für ihr Wirtschaftsmodell lebenswichtigen russischen Subventionen fürchten.

\section{Auswirkungen der ukrainischen Proteste}

Die anhaltenden Proteste in der Ukraine, deren Forderung nach Unterzeichnung des Assoziationsabkommens sich längst zu weitergehenden politischen Forderungen nach Regierungsrücktritt und Neuwahlen erweitert hat, verändern auch die Bewertungen der Ereignisse in und für Belarus. Während belarussische Oppositionspolitiker nach Kiew reisen, um zumindest an den Revolutionen anderer Völker teilhaben zu können, dürfte sich die belarussische Staatsspitze in ihrem Vorgehen bestätigt sehen, durch eine repressive Politik gefährliche Protestansätze bereits im Keim zu ersticken. Die ukrainischen Proteste führen zudem der EU wieder einmal die Duldsamkeit der belarussischen Gesellschaft vor Augen, die sich im Unterschied zur ukrainischen mehrheitlich mit den in ihrem Land herrschenden Verhältnissen abfindet bzw. diese akzeptiert. Dieser Kontrast könnte dazu beitragen, dass die EU zukünftig den politischen Status quo im Lande als langfristig anerkennt. Konsequenz einer solchen Wahrnehmung könnte die Intensivierung eines um Einbeziehung staatlicher Akteure bemühten Ansatzes der EU-Politik gegenüber Belarus sein. Alternativ könnte die EU jedoch auch jegliche aktive Bemühungen um eine Veränderung der Situation aufgrund ihrer vermeintlichen Aussichtslosigkeit einstellen.

\section{Belarussisches Verhandlungsangebot}

Mit der von Außenminister Makej beim Gipfel verkündeten Botschaft, dass Belarus nun bereit sei, Verhandlungen über ein Visaerleichterungsabkommen mit der EU zu beginnen, scheint die belarussische Führung daher auch verhindern zu wollen, dass das Land von der EU-Agenda verschwinden könnte. Eine Lösung der aus EU-Sicht entscheidenden Frage der Freilassung aller politischen Gefangenen ist jedoch nach wie vor nicht in Sicht. Hinter dem Angebot ist gleichwohl das Interesse der belarussischen Führung zu erkennen, den eigenen außenpolitischen Handlungsspielraum zu erweitern und Zugänge zu finanziellen Ressourcen zu sichern, um die bedrohliche Ausmaße annehmende Abhängigkeit des Landes von Russland zu vermindern. Dies gilt umso mehr, als die belarussische Führung die Rache Putins für Lukaschenkas Verhalten während des Kali-Konflikts fürchten muss (vgl. Belarus-Analysen Nr. 14, <http://www.laen der-analysen.de/belarus/pdf/BelarusAnalysen14.pdf >). $\mathrm{Zu}$ den vorbeugenden Maßnahmen gehört auch die Verbesserung der Beziehungen mit dem Westen.

\section{Zwischenbilanz des Kali-Konflikts}

Bereits eine Woche vor dem Gipfel - und fast gleichzeitig mit der Entscheidung von Viktor Janukowitsch, das Assoziationsabkommen mit der EU nicht zu unterzeichnen - lieferte Belarus zudem endlich den im August in Minsk verhafteten Generaldirektor von »Uralkali« Wladislaw Baumgertner nach Russland aus. Zwar hält sich Moskau bisher an die Absprachen hinsichtlich der weiteren Strafverfolgung Baumgertners in Russland - selbst seine am 10. Dezember erfolgte Überführung in den Hausarrest wurde mit Verweis auf die Anwendung dieser Haftform im Falle Baumgertners in Belarus begründet. Von der durch Aljaksandr Lukaschenka noch im Oktober 2013 lauthals erhobenen Forderung, dass Belarus im Falle der Auslieferung Baumgertners 100 Mio. US-Dollar Schadenersatz für die infolge des Austritts von »Uralkali« aus dem Belarussischen Kali-Kartell entstandenen wirtschaftlichen Verluste erhalten müsse, ist jedoch keine Rede mehr. Unklar ist auch, ob die neuen Einzelaktionäre von »Uralkali« Michail Prochorow und Dmitrij Masepin ein reales Interesse an einer Wiederherstellung des gemeinsamen Kartells haben. Zwar handelt es sich bei dem Besitzer von "Uralchim" Masepin um einen gebürtigen Belarussen, doch hatte Lukaschenka mehrmals öffentlich den Wunsch geäußert, der Präsident von "Rosneft « Michail Guzeriew möge das Aktienpaket von Sulejman Kerimow aufkaufen. 


\section{Wachsende Liquiditätsprobleme}

Belarus versucht daher zunehmend zu demonstrieren, dass es nicht unbedingt auf die Kooperation mit Russland angewiesen ist und sich der Kaliexport wieder positiv entwickle. So ist offiziellen Angaben zufolge der Export von Kalidünger von 350.000 t im September auf 420.000 t im Oktober 2013 gestiegen. Im Oktober 2013 wurde zudem ein dreijähriges Lieferabkommen mit dem chinesischen Konzern »Sinochem« unterzeichnet. Selbst wenn die jüngsten Exportangaben korrekt sein sollten, ist das belarussische Exportgeschäft infolge des gesunkenen Weltmarktpreises für Kalidünger hart getroffen. Für die Außenhandelsbilanz kommt erschwerend hinzu, dass Russland seine Öllieferungen an Belarus im zweiten Halbjahr reduziert, so dass Belarus nur 19,25 Mio. $t$ anstelle der erwarteten 23 Mio. $t$ bis zum Jahresende erhalten wird. Auch die Auszahlung der letzten Tranche des Kredits der Eurasischen Entwicklungsbank ist bisher nicht erfolgt, und ein neuer russischer Kredit ist nicht in Sicht.

Der auf offizieller belarussischer Seite zunehmend geäußerte Wunsch, die Beziehungen mit dem Westen zu verbessern, ist nicht zuletzt vor dem Hintergrund der wachsenden finanziellen Liquiditätsprobleme des Landes zu sehen. Mit einem im Oktober 2013 aufgelegten Plan für strukturelle Wirtschaftsreformen machten Regierung und Nationalbank deutlich, dass sie Ambitionen auf einen neuen Kredit des Internationalen Währungsfonds verfolgen. Hierin treffen sie sich mit der ukrainischen Führung, seitdem diese den Erhalt von finanzieller Unterstützung durch die EU und den IWF zur Voraussetzung für die Unterzeichnung des Assoziationsabkommens zu einem späteren Zeitpunkt erklärt hat. Der Eindruck, dass die Führungen beider Länder, ihre integrationspolitische Wahl quasi meistbietend zu verkaufen beabsichtigen, könnte das Engagement für eine aktive Ostpolitik innerhalb der EU weiter reduzieren.

\section{Langsamer Wertewandel}

Angesichts des in Deutschland und der EU bereits seit längerem dominierenden Eindruck, dass die Politik gegenüber Belarus sich in einer Sackgasse befinde und das bisherige Engagement praktisch keine Effekte erzielt habe, besteht die Gefahr zu übersehen, dass sich auch ohne Massenproteste in Belarus ein allmählicher Wertewandel vollzieht. Deutlich erkennbar ist dies beispielsweise an den in diesem Jahr durchgeführten Umfragen zur Todesstrafe (siehe Grafiken in dieser Ausgabe). Denn die Umfragen zeigen, dass die beim umstrittenen Verfassungsreferendum im November 1996 vorhandene Mehrheit von $80,4 \%$ zur Beibehaltung der Todesstrafe, auf die sich Präsident Lukaschenka in seinen Stellungnahmen zur Todesstrafe bis heute bezieht, in dieser Form nicht mehr existiert. Zwar ist die Zahl der Befürworter weiterhin in der Mehrheit, doch immerhin 44,5\% der Befragten sprechen sich für eine sofortige bzw. schrittweise Abschaffung der Todesstrafe oder ein Moratorium aus. Die Umfrage zeigt darüberhinaus, dass die Dorfbevölkerung in dieser Frage weitaus offener ist als die Bewohner von Bezirksund Gebietsstädten. Auch die Religiösität scheint eine Rolle zu spielen. Unter gläubigen Personen findet sich der höchste Anteil derer, die sich für eine Abschaffung oder Einschränkung der Todesstrafe aussprechen.

\section{Rolle der Kirchen}

Diese Haltung spiegelt sich auch in den öffentlich geäußerten Positionen der Oberhäupter der katholischen und der orthodoxen Kirche wider. Metropolit Filaret hatte bereits beim Referendum 1996 die Belarussen aufgefordert, für die Abstimmung der Todesstrafe zu stimmen. Auf diese Position bezog er sich auch beim Runden Tisch "Religion und Todesstrafe«, den die belarussische Regierung im Juni 2013 in Kooperation mit dem Europarat in Minsk durchführte. Im Falle der vermeintlichen UBahn-Attentäter Uladislau Kawaljou und Dsmitrij Kanawalau, die im März 2012 hingerichtet wurden, hatte sich Metropolit Filaret jedoch im Unterschied zu Erzbischof Kondrusiewicz nicht öffentlich für eine Begnadigung eingesetzt. Während Kondrusiewicz Präsident Lukaschenka direkt aufrief, ein Moratorium auf die Ausübung der Todesstrafe zu verhängen, veröffentlichte Filarets Pressestelle im Dezember 2011 eine Erklärung, in der es hieß: „Die Kirche darf nicht kategorisch die Abschaffung der Todesstrafe fordern - insbesondere in einer Gesellschaft, in der die Mehrheit so entschlossen daran festhält.« Anfang Dezember 2013 schrieb Metropolit Filaret jedoch einen öffentlichen Brief an die Mutter eines wegen Mordes zu Tode verurteilten Mannes, indem er erneut betont, dass die orthodoxe Kirche sich dafür einsetze, dass die Todesstrafe nicht zur Anwendung komme. Gleichzeitig wurde das Schreiben beim Begnadigungsauschuss der Präsidialadministration eingereicht.

\section{Gefahr wirtschaftlicher Engführung}

In Übereinstimmung mit der Position der Kirchen zur Todesstrafe ist auch, dass sich die zunehmend kritische Haltung zur Todesstrafe wesentlich auf der Angst vor Fehlurteilen gründet. Das Argument, ein Moratorium sei von Vorteil im Hinblick auf einen eventuellen EU-Beitritt, ist hingegen nur äußerst selten zu finden. Das Thema Todesstrafe wird damit in der gesellschaftlichen Haltung nicht außenpolitisch instrumentalisiert. Auch hieraus lassen sich Anregungen für die Frage ziehen, wie die EU ihre zukünftige Politik gegenüber Belarus und den anderen östlichen Nachbarstaaten gestalten soll. Denn der Entwicklung von vielfältigen, nicht an konkreten Kon- 
ditionen gebundenen Dialog- und Kooperationsformen kommt offensichtlich eine wichtige Rolle beim allmählichen Wertewandel in der belarussischen Gesellschaft zu. Es sollte daher keine einseitige Zuspitzung der Diskussion um die zukünftige EU-Politik gegenüber ihren östlichen Nachbarstaaten auf die Frage erfolgen, ob die EU ausrei- chend wirtschaftliche Anreize und finanzielle Unterstützung bietet - so richtig und wichtig das von praktisch allen nachfolgenden belarussischen Experten vertretene Argument ist, dass die EU derzeit keine Instrumente hat, um dem russischen Angebot kurzfristig spürbarer Subventionen etwas Vergleichbares entgegenzustellen.

Lesetipps:

zum EU-Gipfel in Vilnius

- Maria Davydchyk: Belarus nach dem Gipfeltreffen in Vilnius: Bilanz und Perspektiven, in GET Belarus Newsletter, Nr. 25, November-Dezember 2013, <http://www.get-belarus.de/download/Newsletter/2013/Newsletter_25_2013_ GET\%20Belarus.pdf?PHPSESSID=e3b4bdaf60fb65211470c8d13038c85e>

zur Position der Kirchen gegenüber der Todesstrafe

- Mitropolit Filaret: »My, christiane, ne moshem oprawdywat smertnuju kasn« [Metropolit Filaret: "Wir Christen können die Todesstrafe nicht rechtfertigen«], 21.06.2013, <http://www.church.by/ru/node/1086>

- Christanskoe otnoschenie k probleme smertnoj kasni [Die christliche Haltung zum Problem der Todesstrafe], 13.12.2011, <http://www.church.by/resource/Dir0301/Dir0302/2011/Page3998.html>

- Mitropolit Kandrusewitsch swjartaezza da uldau Belarusi s saklikam uweszi maratoryj na smjarotnae pakaranne [Metropolit Kondrusiewicz ruft die belarussische Führung auf, ein Moratorium für die Todesstrafe einzuführen], 2.12.2013, <http://catholic.by/2/libr/interview/110860-kandrusiewicz.html>

\section{Bewertungen des EU-Gipfels in Vilnius durch belarussische Experten}

\section{Zusammenfassung:}

Die nachfolgenden Beiträge unabhängiger belarussischer Experten geben Anwort auf folgende Fragen: a) Gesamtbewertung der Ergebnisse des EU-Gipfels in Vilnius, b) Auswirkungen des Scheitern des EU-Assoziationsabkommens mit der Ukraine für Belarus, c) Bewertung des Verhandlungsangebots des belarussischen Außenministers Uladsimir Makej über ein Visaerleichterungsabkommen mit der EU, d) nach den Möglichkeiten zivilgesellschaftlicher Vertreter, ihre Positionen im Vorfeld und während des Gipfels zu präsentieren sowie e) nach den Perspektiven der "Östlichen Partnerschaft«. Insgesamt zeigt sich darin eine hohe Skepsis gegenüber dem multilateralen Ansatz der EU sowie das Plädoyer für eine Verstärkung des finanziellen Engagements der EU in der Region.

\section{Multilateraler Ansatz der Östlichen Partnerschaft ohne Perspektiven}

Denis Meljanzau, Vilnius

\section{Vorzeigbare Ergebnisse}

Der Gipfel ist für Litauen sehr erfolgreich verlaufen, wenn man sich die Anzahl der teilnehmenden Staatsund Regierungschefs sowie die hohe Aufmerksamkeit der europäischen Medien anschaut. Auf dem Gipfel wurden nicht nur Integrationsfortschritte mit den aktiven Partnern Georgien und Moldowa fixiert, sondern es konnten auch einige Erfolge in den Beziehungen zu den Outsidern der "Östlichen Partnerschaft « vorgezeigt werden: mit Aserbaidschan wurde das Abkommen über die Erleichterung der Ausstellung von Visa unterzeichnet und der belarussische Außenminister verkündete die Bereitschaft seines Landes, Verhandlungen über ein Visaerleichterungsabkommen mit der EU zu beginnen. 
Selbstverständlich sollte die Unterzeichnung des Assoziationsabkommens mit der Ukraine der Höhepunkt des Gipfels werden, und sein Scheitern hat den Gipfel überschattet. Nichtsdestotrotz hat der Gipfel gezeigt, dass die Östliche Partnerschaft einige konkrete Ergebnisse vorzuweisen hat.

\section{Das gescheiterte Ukraine-Abkommen}

Die Entscheidung der Ukraine, das Abkommen mit der EU jetzt nicht zu unterzeichnen, sollte aus meiner Sicht nicht als endgültiges Scheitern der europäischen Integration der Ukraine angesehen werden. Der ukrainische Präsident hat betont, dass die Verhandlungen fortgesetzt werden sollen und eine Unterzeichnung in der Zukunft möglich sei. Für Belarus hätte ein Beitrittsbeschluss der Ukraine zur Zollunion in der Tat ernsthafte Auswirkungen. Denn in diesem Falle würde die Ukraine zum direkten Konkurrenten um die begrenzten russischen Subventionen. Belarus würde seine bisherige Bedeutung als Schlüsselpartner für die Integrationspläne Russlands verlieren. Gleichzeitig hätte die Zuwendung der Ukraine Richtung Russland wahrscheinlich auch eine veränderte Positionierung der EU gegenüber der Region zur Folge: Das belarussische Regime wäre nicht länger das einzige schwarze Schaf und Brüssel müsste aktiver nach neuen Kooperationsstrategien mit den integrationsunwilligen postsowjetischen Ländern Osteuropas suchen, bei denen das Prinzip »more for more« nicht wirkt.

\section{Das belarussische Verhandlungsangebot}

Die Ankündigung des belarussischen Außenministers Uladsimir Makej, dass Belarus nun bereit sei, Verhandlungen über ein Visaerleichterungsabkommen mit der EU aufzunehmen, ist aus meiner Sicht positiv zu bewerten. Seitdem Belarus und Russland Mitte November 2013 ein Rückübernahmeabkommen unterzeichnet haben, gibt es keine formalen Hindernisse mehr, die den Beginn entsprechender Verhandlungen erschweren würden. Man sollte auch in Erinnerung behalten, dass es die belarussische Führung war, die der Europäischen Kommission bereits 2004 den Beginn von Visaerleichterungsverhandlungen angeboten hatte, doch damals war die EU aus politischen Gründen hierzu nicht bereit. In 2011 hat die EU ihre Position in dieser Frage jedoch um 180 Grad gedreht.

\section{Zivilgesellschaftliche Akteure}

Zivilgesellschaftliche Organisationen hatten zahlreiche Möglichkeiten ihre Positionen zur Östlichen Partnerschaft und anderen relevanten Themen darzustellen und haben diese Möglichkeiten auch genutzt. Unter den Veranstaltungen, die vor und während des Gipfels stattfanden, sind hervorzuheben: das Zivilgesellschaftliche Forum der Östlichen Partnerschaft Anfang Oktober in Moldowa, das Belarussische Menschenrechtsforum und die Minsk-Forum-Konferenz "Strategies for Future Policy Towards Belarus« Ende Oktober in Vilnius sowie die Konferenz »Eastern Partnership Reality Check« während des Gipfels ebenfalls in Vilnius.

\section{Perspektiven}

Ein zentrales Problem der "Östlichen Partnerschaft" besteht darin, dass die EU ein einheitliches Format in einer heterogenen Region anzuwenden versucht. Bei den Ländern der "Östlichen Partnerschaft« handelt es sich um Staaten mit unterschiedlichen politischen Regimen und Wirtschaftssystemen, die sich zudem verschiedenen geographischen Regionen zugehörig fühlen. Auch ihr Interesse an der Integration mit der EU ist höchst unterschiedlich ausgeprägt. Mit anderen Worten: Die Länder haben nur wenig gemeinsam und deswegen ist das multilaterale Kooperationsformat eher ein künstliches. Hinzu kommt, dass die EU selber die deklarierte einheitliche Vorgehensweise nicht durchgängig einhält. So sind die Beziehungen zum autoritären Regime von Alijew beispielsweise wesentlich besser als die mit dem vergleichsweise "weicheren « Regime in Belarus. Die EU sollte deswegen mehr Zeit dafür aufbringen, individuelle Zugänge zu den Partnerländern zu entwickeln, und eine mehr pragmatische Position gegenüber den Staaten beziehen, welche die Werte der EU nicht zu den ihrigen machen. Ansonsten wird es schwierig sein, von Aussichten auf konstruktive, gutnachbarschaftliche Beziehungen zu sprechen.

\section{Über den Autor:}

Denis Meljanzau ist Direktor des Belarussischen Instituts für Internationale Strategische Studien in Vilnius (<http:// belinstitute.eu>).

\section{Lesetipp:}

Dzianis Melyantsou, What should the EU do with Belarus? In: BelarusInfoLetter, hrsg. vom Eastern Europe Studies Centre in Vilnius, 6/2013, S. 1-3, <http://www.eesc.lt/uploads/news/id521/Bell\%202013_6\%2836\%29.pdf> 


\title{
Drohende Demoralisierung der Zivilgesellschaft
}

\author{
Aljaksandr Adamjanz, Minsk
}

$\mathrm{D}$ e Ergebnisse des EU-Gipfels haben aus meiner Sicht für Belarus nichts Konkretes ergeben. Das zentrale Ereignis ist das Scheitern des Assoziationsabkommens mit der Ukraine, das eine große Niederlage der EU-Diplomatie darstellt. Viele Experten vertreten die Ansicht, dass mit der Annäherung der Ukraine an Russland das Interesse der russischen Führung an Belarus nachlasse, bzw. umgekehrt, dass das Interesse an Belarus nur so lange hoch bleibt, wie die Annäherung der Ukraine an Russland noch eine offene Frage ist. Daraus wird gefolgert, dass die Rückkehr der Ukraine in die russische Einflusssphäre für die Demokratisierung von Belarus von Vorteil sei. Diese Annahme, die sich vielfach in den Medien lesen lässt, greift meines Erachtens jedoch zu kurz.

Meiner Einschätzung nach hat der Misserfolg der EU, die Ukraine eng an die Europäische Union zu binden, vorrangig negative Auswirkungen für Belarus. Denn das Scheitern der europäischen Integration der Ukraine stellt einen Demoralisierungsschub für die demokratischen Kräfte in Belarus dar, während die Unterzeichnung des Assoziationsabkommens der belarussischen Zivilgesellschaft einen starken moralischen Impuls gegeben hätte. Letzteres gilt auch für alle anderen Länder der Östlichen Partnerschaft und für Russland. Kurzfristig hätten die politischen Führungen in den autoritären Nachbarstaaten in Reaktion auf die ukrainische EU-Annäherung sicher die Daumenschrauben angezogen und den Druck auf die zivilgesellschaftlichen Organisationen verstärkt. Dennoch hätte sich die Situation in den Ländern der Östlichen Partnerschaft grundlegend verändert und die autoritären Regime wären mittelfristig geschwächt worden. Angesichts der sich verschlechternden wirtschaftlichen Situation in Russland, die sich auch auf Belarus auswirkt, hätte der endgültige Verlust der Ukraine für den Kreml einen harten Schlag bedeutet, von dem er sich nicht mehr richtig erholt hätte.

Die EU konnte den Erfolg des Assoziationsabkommens nicht sichern, weil ihre Politik der enormen Bedeutung der Ukraine für die Ostpolitik der Union sowie für den gesamten postsowjetischen Raum nicht gerecht wurde. Ebenso wurden die russischen Einflussmöglichkeiten unterschätzt. Die EU-Politik müsste daher proaktiver sein und über ein besser ausgestattetes finanzielles Fundament verfügen. In der Realität werden für die Initiative "Östliche Partnerschaft« nur geringe Finanzmittel zur Verfügung gestellt, die keinen signifikanten Anreiz darstellen können.

Über den Autor:

Aljaksandr Adamjanz ist Direktor des Zentrums für europäische Studien in Minsk (<http://eurocenter.by>).

Lesetipp:

Aljaksandr Adamjanz: Polemika. Konserwatiwnaja rewoljuzija: rywok v proschloe [Polemik. Die konservative Revolution als Sprung in die Vergangenheit], 14.03.2013, <http://naviny.by/rubrics/opinion/2013/03/14/ic_articles_410_181125/>

\section{Die ukrainische Krise als Ausdruck des Scheiterns der EU-Außenpolitik}

Andrej Kasakewitsch, Minsk

$\mathrm{D}$ er Gipfel in Vilnius hat das Scheitern der EUAußenpolitik und der Initiative "Östliche Partnerschaft« in der Zielregion offensichtlich gemacht. In den Monaten vor dem Gipfel waren die meisten Experten und Politiker davon überzeugt, dass beim Gipfel eine Unterzeichnung des Assoziationsabkommens mit der Ukraine erfolgen wird. Doch die Position der Ukraine hat sich verändert, und die europäischen Strukturen haben sich sowohl als unfähig erwiesen dies vorherzusehen als auch auf diesen Prozess Einfluss zu nehmen.

\section{Konsequenzen für Belarus}

Wie sich der ukrainische Faktor auf Belarus auswirkt, kann derzeit noch nicht endgültig eingeschätzt werden, da sich die Situation in der Ukraine ständig verändert und vieles davon abhängt, wie die innenpolitische Krise in der Ukraine gelöst wird - wobei die EU hier faktisch als Vermittler fungiert. Aus verschiedenen wirtschaftlichen Gründen wäre die Unterzeichnung des Abkommens durch die Ukraine für Belarus nicht von Vorteil gewesen. Mit hoher Wahrscheinlichkeit hätte die belarussische Volkswirtschaft einige Absatzmärkte verloren 
und in einigen Branchen, wie im Internationalen Logistik-Bereich, an Konkurrenzfähigkeit eingebüßt. Diese Probleme entfallen nun. Hingegen kann die Einbeziehung der Ukraine in das »eurasische« Integrationsprojekt in der jetzigen Situation für Belarus praktische Vorteile bringen, da sich hierdurch der Handlungsspielraum für die eigene Interessenvertretung in Moskau vergrößert.

In strategischer Hinsicht dürfte der Effekt jedoch ein negativer sein. Denn die Ukraine wird einen beträchtlichen Teil der Aufmerksamkeit und der finanziellen Ressourcen binden, wodurch sich automatisch die Bedeutung von Belarus für Russland verringern wird. Als Folge hiervon dürften sich die Beziehungen zwischen Belarus und Russland weiter verschlechtern. Aber es muss nochmals betont werden, dass die weitere Entwicklung von dem Ausgang der Ereignisse in der Ukraine abhängt.

\section{Wirtschaftsfragen als Schlüssel}

Mit dem Angebot von Außenminister Makej, mit der EU Verhandlungen über ein Visaerleichterungsabkommen aufzunehmen, hat Belarus beim EU-Gipfel in Vilnius die Bereitschaft zu einem begrenzten Dialog mit der Europäischen Union demonstriert. Dieses Verhalten ist nichts grundsätzlich Neues oder Unerwartetes. Zudem war diese Frage nur ein Randthema auf dem Gipfel. Angesichts der anhaltenden globalen Wirtschaftskrise müssen insgesamt die wirtschaftlichen Komponenten der Initiative gestärkt werden, um die Effektivität der Initiative "Östliche Partnerschaft« zu verbessern. Gleichzeitig muss die EU der politischen Situation in der Gesamtregion mehr Aufmerksamkeit widmen, insbesondere dem Einfluss Russlands und anderer großer Staaten.

Der multilaterale Ansatz kann die Entwicklung von bilateralen Beziehungen mit einzelnen Staaten nicht ersetzen, sondern ihnen nur eine zusätzliche Dimension geben. Es wäre ein Fehler, Russland als dritten Akteur in die Beziehungen zwischen der EU und den Ländern der Region einzubeziehen - vor allem weil Russland sein eigenes Integrationsprojekt in Gestalt der Zollunion als Gegengewicht zur EU verfolgt. Dieses Projekt ist vor allem politisch motiviert, so dass die EU im Falle der Einbeziehung Russlands ihre Politik gegenüber den östlichen Nachbarstaaten von den politischen Ambitionen und Interessen Russlands abhängig machen würde.

Über den Autor:

Andrej Kasakewitsch, Politikwissenschaftler, ist Direktor des Instituts für politische Studien in Minsk und Herausgeber der politikwissenschaftlichen Zeitschrift »Polititscheskaja sfera« (<www.palityka.org $>$ ).

Lesetipp:

"Andrej Kasakewitsch: Nelsja skasat chto Belarus idjot swoim putjom" [Andrej Kasakewitsch: Es ist nicht korrekt zu sagen, dass Belarus seinen eigenen Weg geht], in: Swobodnye nowosti-pljus, 9.9.2013, <http://www.sn-plus.com/ru/ page/mainevents/3249/>

\section{Zunehmende Integrationskonkurrenz zwischen Brüssel und Moskau als Chance für Belarus?}

Walerij Karbalewitsch, Minsk

\section{Gemischte Bilanz}

Der EU-Gipfel in Vilnius ist insgesamt erfolgreich erlaufen, doch sind die Probleme unübersehbar. Von den vier Staaten, mit denen die Integration vertieft werden sollte, haben nur Georgien und Moldowa die ausgehandelten Assoziationsabkommen paraphrasiert. Armenien erklärte hingegen bereits im September - nach einem Gespräch seines Staatsoberhaupts mit dem russischen Präsidenten -, dass es der Zollunion beitreten wolle. Kurz vor dem Gipfel veränderte auch der ukrainische Präsident nach einem Besuch in Moskau seine Position. Dabei ist die ukrainische Entscheidung von größerer Tragweite. Die Entscheidung von Präsident Viktor Janukowitsch hat ein solches politisches Erdbeben im Lande ausgelöst, dass er wahrscheinlich selbst nicht mehr froh ist über seinen Schritt.

Russland hat selbstverständlich alle erdenklichen Hebel benutzt, um die Ukraine Richtung Osten zu wenden. Doch warum verfügte Moskau über mehr Einflussmöglichkeiten als Brüssel? Die Initiative »Östliche Partnerschaft«, die 2009 aufgelegt wurde, setzt auf die gleiche Logik, welche die EU bereits in ihrer Erweiterungspolitik angewandt hat und die sich in der Formel "more for more« ausdrückt. D. h. je tiefgreifender politische und wirtschaftliche Reformen zur Umsetzung europäischer Standards von den postsowjetischen Staaten durchgeführt werden, desto intensiver soll die Unterstützung von Seiten der Europäischen Union ausfallen. 


\section{Defizite der EU-Politik}

Dies ist ein schönes Konzept. Doch der Gipfel in Vilnius hat gezeigt, dass die Realität eine andere ist. Das Prinzip, das gegenüber den Ländern Mitteleuropas und des Baltikums hervorragend funktioniert hat, erweist sich gegenüber den Mitgliedern der "Östlichen Partnerschaft« als ineffektiv. Hierfür gibt es mehrere Gründe. Vor allem ist der Lohn, den die EU diesen Ländern für ihre Mühen bietet, zu klein. Ihnen wird keine vollwertige Mitgliedschaft angeboten, sondern lediglich unterschiedliche Kooperationsformen. Das Fehlen der Beitrittsperspektive hätte mit Geld kompensiert werden können. Westliche Experten erwähnen oft, dass die finanzielle Unterstützung durch den amerikanischen Marschall-Plan nach dem Zweiten Weltkrieg eine wichtige Rolle gespielt hat, um die Expansion der Sowjetunion einzudämmen. Die reichere EU könnte in dieser Hinsicht leicht Russland als Konkurrenten um die Kontrolle in Osteuropa und im Kaukasus ausschalten.

Das Problem besteht darin, dass die EU nicht bereit ist, bedeutende Ressourcen in das Projekt "Östliche Partnerschaft« zu investieren. Im vergangenen Jahr standen für alle sechs Partnerländer insgesamt 600 Mio. Euro zur Verfügung. Zum Vergleich: Allein Belarus hat in 2012 von Russland etwa 10 Mrd. US-Dollar erhalten. Eine Ursache hierfür ist die Wirtschaftskrise in der EU, die sie zwingt viel Geld für die Rettung einzelner Mitgliedsstaaten, wie Griechenland, aufzubringen. Zudem hat die EU keine Russland vergleichbaren imperialen Ambitionen, welche einen hohen Mitteleinsatz legitimieren würden. In den EU-Ländern wird der Staatshaushalt im Unterschied zu Russland durch die Gesellschaft mittels des Parlaments kontrolliert. Und die Wähler in Portugal oder Irland verstehen nicht, warum ihr Geld für irgendwelche geopolitischen Projekte in weit entfernten Ländern verwendet werden soll.

\section{Fisch statt Angel}

Die EU setzte darauf, die Länder der »Östlichen Partnerschaft« allein aufgrund der Attraktivität ihres sozialen Modells europäisieren zu können - nach dem Prinzip, im Unterschied zu Russland geben wir Euch keinen Fisch, sondern eine Angel. Sie hat damit gerechnet, dass die Perspektive auf zukünftigen Wohlstand sich als gewichtiger erweisen würde als die jetzt greifbaren russischen Subventionen. Doch diese Rechnung ist nicht aufgegangen, da das europäische Sozialmodell angesichts der Wirtschaftskrise in Europa viel von seiner Attraktivität verloren hat. Hinzu kommt, dass die postsowjetischen Gesellschaften mental weniger auf die Marktwirtschaft und die Demokratie vorbereitet waren als die Menschen in Mitteleuropa und im Baltikum. Für die politischen Führungen ist es einfacher ohne Demokratie zu regieren und sie bevorzugen autoritäre Methoden. Aber auch die Menschen sind nicht bereit, eine neue Lebensweise anzunehmen - wobei ich mich freuen würde, wenn die Proteste auf dem Kiewer Maidan diese These wiederlegen würden. Denn alle wirtschaftlichen und politischen Reformen sind stets schmerzhaft und mit psychischem Stress verbunden. EU-Hilfe ist freilich nur mit Veränderungen zu haben, während Russland in der Regel nicht für Reformen, sondern für die Erhaltung des Status quo zahlt.

\section{Ukraine als Konkurrent}

Die ukrainische Entscheidung, das Assoziationsabkommen mit der EU nicht zu unterschreiben, hat in mehrfacher Hinsicht Auswirkungen für Belarus:

1. Hätte die EU beim Gipfel in Vilnius alle geplanten Abkommen mit Armenien, Georgien, Moldowa und der Ukraine realisieren können, dann wäre beim Gipfel sehr sichtbar gewesen, dass Belarus nicht an den zentralen europäischen Prozessen beteiligt ist. Die Kehrtwende von Armenien und der Ukraine hatte jedoch zur Folge, dass die Außenseiterposition von Belarus nicht derart offensichtlich war. Lukaschenka kann nun vielmehr behaupten, dass er stets konsequent gewesen sei und im Unterschied zu anderen Staatschefs keinen Zick-Zack-Kurs betreibe. Dies ist für das offizielle Minsk ein Plus.

2. Russland muss nun Armenien und der Ukraine deutlich mehr wirtschaftliche Unterstützung erweisen, um beide Länder in seinem Einflusskreis zu halten. Damit verschärft sich die Konkurrenz um russische Ressourcen. Da die Ukraine für Russland eine höhere außenpolitische Bedeutung hat, könnten sich die russischen Subventionen für die belarussische Wirtschaft verringern.

3. Die Proteste auf dem Majdan in Kiew geben ein Beispiel, wie die Bürger eines Nachbarstaates Druck auf ihre Politiker ausüben. Lukaschenka hat große Furcht vor Straßenprotesten. Darin liegt eine weitere negative Auswirkung der aktuellen Ereignisse.

Im Unterschied zum EU-Gipfel in Warschau vor zwei Jahren hat das offizielle Minsk dieses Jahr in Vilnius für keinen Skandal gesorgt. Zur Erinnerung: Damals hatte die belarussische Delegation, die lediglich vom belarussischen Botschafter in Polen geleitet wurde, den Gipfel demonstrativ vorzeitig verlassen. In diesem Sinne kann man von einem Fortschritt sprechen. Zudem zeugt die Erklärung von Außenminister Makej, dass Belarus nun zu Verhandlungen über ein Visaerleichterungsabkommen bereit sei, davon, dass die belarussische Führung zwar nicht das Fenster öffnet, aber zumindest einen Fensterspalt offen halten möchte.

\section{Fragliche Visaerleichterung}

Allerdings ist die belarussische Seite meiner Ansicht nach nicht an einem raschen erfolgreichen Abschluss dieser 
Verhandlungen interessiert. Denn es ist ihr nicht daran gelegen, dass die belarussischen Bürger mehr Reisemöglichkeiten in die EU erhalten. Dies zeigt sich daran, dass Belarus nichts dafür tut, damit die mit Litauen und Polen vor Jahren unterzeichneten Abkommen über den vereinfachten Grenzverkehr in grenznahen Regionen in Kraft treten können. Litauen und Polen haben bereits alle für die Umsetzung erforderlichen Ratifizierungsverfahren abgeschlossen, Belarus schiebt diesen Prozess jedoch immer wieder auf. Für diese Position lassen sich mehrere Gründe nennen:

1. Die belarussische Führung sieht in der Ratifizierungsverweigerung ein Mittel, mit dem sie die EU insgesamt sowie Litauen und Polen im Besonderen unter Druck setzen kann. Wenn die Nachbarstatten an der Realisierung dieser Abkommen interessiert sind, dann sollen sie ihre Kritik an der Menschenrechtssituation und die Unterstützung für die Opposition in Belarus einstellen sowie die EU-Sanktionen beenden. Nur wenn es ein Entgegenkommen in diesen Fragen gibt, will die belarussische Führung ihre Blockadehaltung aufgeben.

2. Die belarussische Führung ist grundsätzlich nicht an einem Massenexodus ihrer Bürger in Richtung Westen interessiert. Damit teilt sie die Logik aller autoritären Regime, die an eine Festung erinnern und einen eigenen Sonderweg für sich in Anspruch nehmen, um ihre Alleinherrschaft zu sichern. Ihr Immunsystem ist darauf ausgerichtet, alle fremden Einflüsse von außen, welche den Freiheitsvirus in sich tragen, abzuwehren. Aus diesem Grunde werden auch - begleitet von vorgeschobenen gut meinenden Argumenten - die Möglichkeiten einer legalen Beschäftigung im Ausland eingeschränkt, drohen die Behörden mit der Einführung einer Ausreisesteuer in Höhe von 100 US-Dollar "pro Nase« und erschweren die Ausreise von Studenten zum Studium oder von Kindern zur Erholung etc. Denn der Vergleich des Lebensstandards, des Preisniveaus in den Geschäften und vieles andere fallen nicht zum Vorteil der "blühenden Republik Belarus« aus. Und die Führung muss verhindern, dass sich hierdurch das Mas- senbewusstsein umorientiert. Denn die schlimmste Form des Gefängnisses ist die, bei der die Insassen überhaupt nicht ahnen, dass sie im Gefängnis leben.

3. Aleksjandr Lukaschenka und seiner nächsten Umgebung ist bekanntermaßen die Einreise in die EUMitgliedsländer verboten. Aus diesem Grunde gefällt es ihm verständlicherweise nicht, wenn die Bürger seines Landes aktiv reisen können.

4. Eine recht hohe Anzahl von Belarussen fährt ins Ausland, um einzukaufen. In Litauen, Lettland, Polen und der Ukraine sind die Preise für fast alle Waren deutlich niedriger als in Belarus - obwohl sie dort nicht wie bei uns durch den Staat kontrolliert werden. Wenn die Abkommen über den vereinfachten Grenzverkehr in grenznahen Gebieten in Kraft treten, würden die hierdurch begünstigten belarussischen Bürger noch mehr ausländische Waren kaufen. Dadurch würde das Handelsbilanzdefizit als zentrales Problem der belarussischen Wirtschaft weiter verschärft.

\section{Wechselseitige Enttäuschung}

Heute sind die EU und die Staaten der "Östlichen Partnerschaft« offensichtlich wechselseitig voneinander enttäuscht. Meiner Ansicht nach gibt es in der aktuellen Situation drei Handlungsoptionen für Brüssel. Als erstes könnte Brüssel versuchen, diese Staaten zu kaufen, indem es ihnen mehr finanzielle Ressourcen als Russland anbietet. Zweitens könnte die EU ihre Anforderungen senken und z. B. der Menschenrechtsfrage weniger Beachtung schenken. An erste Stelle würden damit geopolitische Überlegungen treten. Die Themen politische Gefangenen, freie Wahlen etc. kämen damit erst dann wieder auf die Agenda, nachdem es gelungen wäre, diese Staaten aus dem Einflussbereichs Russland zu ziehen. Im Falle einer weiteren Zuspitzung der Integrationskonkurrenz zwischen Brüssel und Moskau wäre diese eine durchaus denkbare Option. Am Wahrscheinlichsten ist jedoch, dass die EU ihre Politik nicht ändern wird, sondern eine abwartende Haltung einnimmt. Denn sie hofft darauf, dass die erwarteten positiven Veränderungen, die man durch die "Östliche Partnerschaft« erreichen wollte, einfach nur mehr Zeit brauchen.

Über den Autor:

Walerij Karbalewitsch, Politikwissenschaftler, arbeitet für das Minsker Analysezentrum »Strategija».

\section{Lesetipp:}

- Walerij Karbalewitsch: Rasotscharowanie ... [Enttäuschung ...], in: Swobodnye nowosti-plus, 4.12.203, <http:// www.sn-plus.com/ru/page/diagnosis/3816/>

- Walerij Karbalewitsch: Wisowye peregowory ne rasmorosjat otnoschenie Minska i Brjusselja [Die Visaverhandlungen beenden nicht die Eiszeit in den Beziehungen zwischen Minsk und Brüssel], Belapan, 29.11.2013, <http://udf.by/news/ politic/91088-valeriy-karbalevich-vizovye-peregovory-s-es-ne-razmorozyat-otnosheniya-minska-i-bryusselya.html> 


\title{
Die EU-Politik des »more for more« ist nicht länger angemessen
}

\author{
Andrej Jahorau, Minsk
}

$\mathrm{D}$ er EU-Gipfel in Vilnius war kein herausragendes Ereignis. Seine Ergebnisse können weder als besonders erfolgreich noch als Ausdruck des Scheiterns betrachtet werden. Das Hauptziel, die Unterzeichnung des Assoziationsabkommens mit der Ukraine, wurde zwar nicht erreicht. Doch wurden die Assoziationsabkommen mit Georgien und Moldowa paraphrasiert, das Visaerleichterungsabkommen mit Aserbaidschan sowie einige weitere weniger bedeutende Abkommen unterzeichnet. Dies sind immerhin einige Zeichen der Annäherung der Länder der "Östlichen Partnerschaft" an die EU. In diesen Prozess fügt sich auch die belarussische Erklärung, Verhandlungen über ein Visaerleichterungsabkommen mit der EU zu beginnen, ein.

\section{Fehlendes Konzept}

Insgesamt ist es freilich besorgniserregend, dass die Gipfelteilnehmer weder über genaue Analysen der aktuellen Situation noch über konkrete Vorstellungen über die weitere Gestaltung der "Östlichen Partnerschaft» verfügen. Vorherrschend war die Position, wie bisher weiterzumachen unter Anwendung des Prinzips »Mehr für mehr« sowie unter Beibehalt des formalistischen Verständnisses von Annäherung im Sinne der Übernahme von EU-Standards in die nationale Gesetzgebung, der Kooperationsentwicklung in bestimmten Bereichen (Energie, Infrastruktur, people-to-peopleKontakte) und der Unterzeichnung von Standardabkommen. Dabei ist offensichtlich, dass die "Östliche Partnerschaft" ihre Ziele nicht in vollem Umfange erreicht. Dies zeigt sich an den Kehrtwendungen von Armenien und der Ukraine sowie an den fehlenden Fortschritten im Umgang mit Belarus. Die EU hat den Einfluss Russlands mit seinen antagonistischen strategischen Interessen unterschätzt sowie nicht ausreichend berücksichtigt, wie sehr sich die Länder der »Östlichen Partnerschaft « von den ehemaligen osteuropäischen Beitrittsstaaten unterscheiden. Es ist unmöglich, institutionelle Reformen nach europäischem Vorbild in den Ländern der »Östlichen Partnerschaft« durchzuführen, ohne die innenpolitische Situation in diesen Ländern und die geopolitischen Spiele in der gesamten Region zu berücksichtigen. Sollte die EU diese Aspekte weiter ignorieren, droht der "Östlichen Partnerschaft» eine ernsthafte Krise.

\section{Die ukrainische Kehrtwende}

Die Weigerung der ukrainischen Führung, dass Assoziationsabkommen mit der EU zu unterschreiben, hat

weitgehende Folgen für die gesamte Region. Indem die Ukraine die Entscheidung über eine vertiefte Kooperation mit der EU aufschiebt, verschiebt sie auch die für das Land notwendigen strukturellen Reformen. Gleichzeitig verstärkt sich aufgrund der offenen geopolitischen Wahl die politische Konfrontation zwischen pro-europäischen und pro-russischen Kräften in der Ukraine. Dadurch erhöhen sich auch die Druckmöglichkeiten Russlands auf die ukrainische Führung. Je länger die Ukraine ihre Wahl hinauszögert, um so schwieriger und teurer wird sie in der Zukunft werden.

Für Belarus hat diese Entwicklung politische und wirtschaftliche Folgen. Denn die Ukraine ist nach der EU und Russland der drittwichtigste Handelspartner der Ukraine. Belarus ist daher erstens an wirtschaftlichem Wachstum und politischer Stabilität in der Ukraine interessiert - was eigentlich einen europäischen Entwicklungsweg des Landes impliziert. Zweitens ist die Annäherung der Ukraine an Russland für Belarus nachteilhaft. Denn der Handlungsspielraum in den komplizierten Beziehungen mit Russland verringert sich dadurch, dass Belarus nicht länger die Rolle des einzigen westlichen Bündnisgenossen Russlands spielen und für diese geopolitische Loyalität Dividenden kassieren kann. Mit einer prorussischen Ukraine eröffnen sich vielmehr neue Möglichkeiten für Russland, Druck auf Belarus auszuüben, Subventionen zu kürzen und auf der Privatisierung von Betrieben zu bestehen, die für das russische Kapital von Interesse sind. Drittens befördert der ukrainische Verzicht auf das Assoziationsabkommen die autoritären Tendenzen im Land analog zu den Entwicklungen in Belarus und Russland. Dadurch werden die pro-europäischen und reformorientierten Kräfte in Belarus weiter geschwächt.

\section{Erforderliche Neujustierung}

Um ihre Initiative "Östliche Partnerschaft" weiterzuentwickeln, sollte die EU an ihrem Instrumentarium arbeiten. Über den jeweiligen Instrumenteneinsatz sollte in Abhängigkeit davon entschieden werden, ob dieser geeignet ist, die Länder der "Östlichen Partnerschaft" in den Orbit der europäischen Transformationsprozesse zu ziehen. Dies bedeutet auch einen partiellen Verzicht auf das Prinzip "more for more«, da dieses den Grad der Annäherung an die EU ausschließlich von den Entscheidungen der regierenden politischen Führungen in den jeweiligen Ländern abhängig macht. Die ukrainische Situation zeigt deutlich, dass politische Führung und Gesellschaft entgegensetzte Orientierungen verfolgen. 
Deshalb könnte die EU hierauf angemessen antworten, indem sie den Handlungen der Regierung "weniger" und den Anliegen der Gesellschaft "mehr« Unterstützung gibt. Eine solche Vorgehensweise würde allerdings eine erhebliche Politisierung der Initiative »Östliche Partnerschaft« bedeuten, die EU-Akteure müssten sich mehr mit der innenpolitischen Situation auseinandersetzen und die Unterstützung der proeuropäischen Kräfte in den Ländern der "Östlichen Partnerschaft« ausdehnen.

Das multilaterale Format der "Östlichen Partnerschaft" sollte beibehalten und weiterentwickelt werden. Denn die Länder der Region und die EU haben viele gemeinsame infrastrukturellen Interessen in den Bereichen Verkehr, Energie, Umwelt etc. Zudem können viele
Probleme, wie die sogenannten "eingefrorenen« regionalen Konflikte, nur gemeinsam gelöst werden. Und schließlich eröffnet es die Möglichkeit, nicht nur die Beziehungen mit der EU, sondern auch untereinander zu entwickeln.

Die EU ist nun in den geopolitischen Konflikt mit Russland in der Region der "Östlichen Partnerschaft" hineingezogen. Die strategische Partnerschaft, welche die EU über lange Jahre mit Russland aufzubauen versuchte, hat sich als nicht tragfähig erwiesen. Es geht daher im Augenblick nicht darum, die Handlungen der EU mit Russland abzustimmen, sondern die Beziehungen der EU mit Russland einer komplexen Analyse und Neubegründung zu unterziehen.

\section{Über den Autor:}

Andrej Jahorau ist Direktor des Zentrums für europäische Transformation (<http://cet.eurobelarus.info $>)$ und nationaler Koordinator des Zivilgesellschaftlichen Forums innerhalb der "Östlichen Partnerschaft».

\section{Lesetipps:}

- Andrei Yahorau und Yury Chauvusau: Dialogue limited to technical and diplomatic level. The Eastern Partnership Roadmap to Vilnius. Belarus: Assessment May 2012 - October 2013, <http:/www.eap-csf.eu/assets/files/Arti cles/Web/Roadmapreports/Belarus\%20roadmap\%20monitoring\%20csf\%20nov\%202013\%20\%283\%29.pdf>

- Andrei Yahorau: The Belarusian Issue in Run-up to the Eastern Partnership Summit in Vilnius, in: BelarusInfoLetter, hrsg. vom Eastern Europe Studies Centre in Vilnius, 6/2013, S. 3-5, <http://www.eesc.lt/uploads/news/ id521/Bell\%202013_6\%2836\%29.pdf>

- Andrei Yahorau: About the tasks of the Country Facilitator, 4.11.2013, <http://eurobelarus.info/en/news/poli tics/2013/11/04/andrei-yahorau-about-the-tasks-of-the-country-facilitator.html>

Übersetzung aller Texte aus dem Russischen von Astrid Sahm 


\section{Öffentliche Meinung zur Todesstrafe}

Die folgenden Meinungsbilder beruhen auf Befragungen, die im April bis Juni 2013 durch SATIO im Rahmen des von »Penal Reform International« geleiteten EU-Projekts »Auf dem Weg zur Abschaffung der Todesstrafe« durchgeführt wurden. Dabei wurden insgesamt 1.100 Personen im Alter von 18 bis 75 Jahren in allen Regionen von Belarus befragt. Die Ergebnisse der Studie wurden im Oktober 2013 durch das Belarussische Helsiniki-Komitee der belarussischen Öffentlichkeit präsentiert.

\section{Grafik 1: Kenntnisstand über die Anwendung der Todesstrafe (\%)}

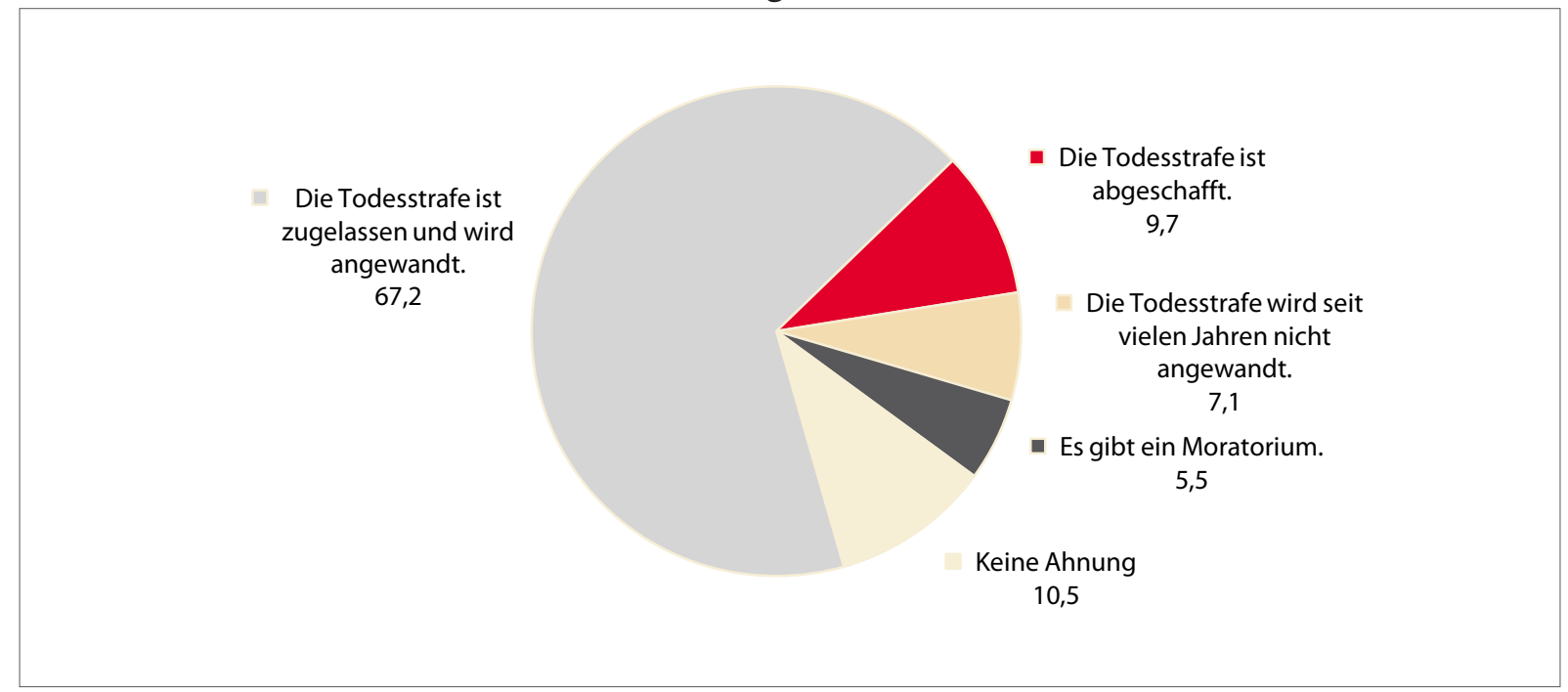

Quelle: A. Akulenko, O. Fablinowa, M. Tschernjanskaja: Prestuplenie i Nakasanie: Wosprijatie, ozenki, otnoschenie obschtschestwa [Verbrechen und Strafen: Wahrnehmung, Bewertungen, Einstellungen der Gesellschaft], Minsk 2013, <http://belhelcom.org/ru/node/18985>, S. 8

Grafik 2: Kenntnisstand über die Anwendung der Todesstrafe in Abhängigkeit vom Wohnort(\%)

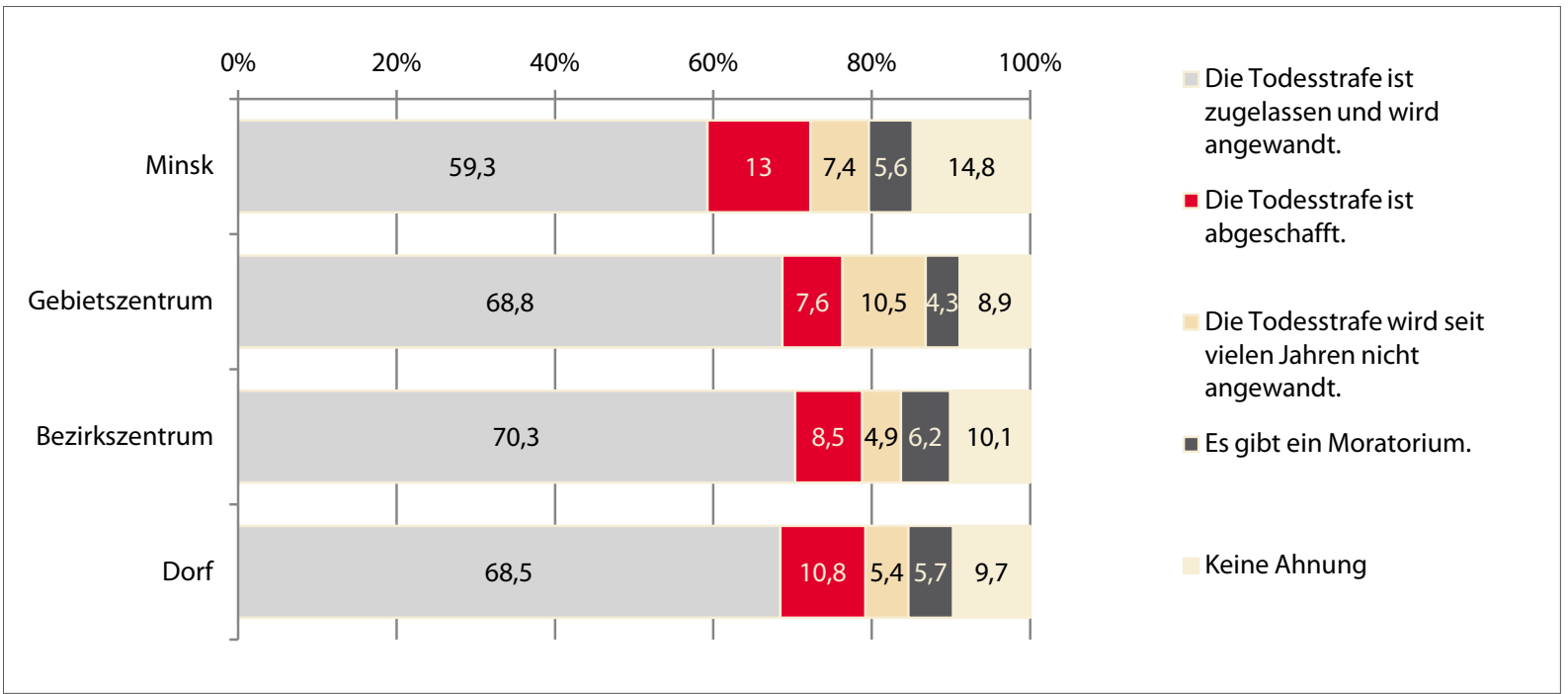

Quelle: A. Akulenko, O. Fablinowa, M. Tschernjanskaja: Prestuplenie i Nakasanie: Wosprijatie, ozenki, otnoschenie obschtschestwa [Verbrechen und Strafen: Wahrnehmung, Bewertungen, Einstellungen der Gesellschaft], Minsk 2013, <http://belhelcom.org/ru/node/18985>, S. 9 


\section{Grafik 3: Einstellung zur Todesstrafe (\%)}

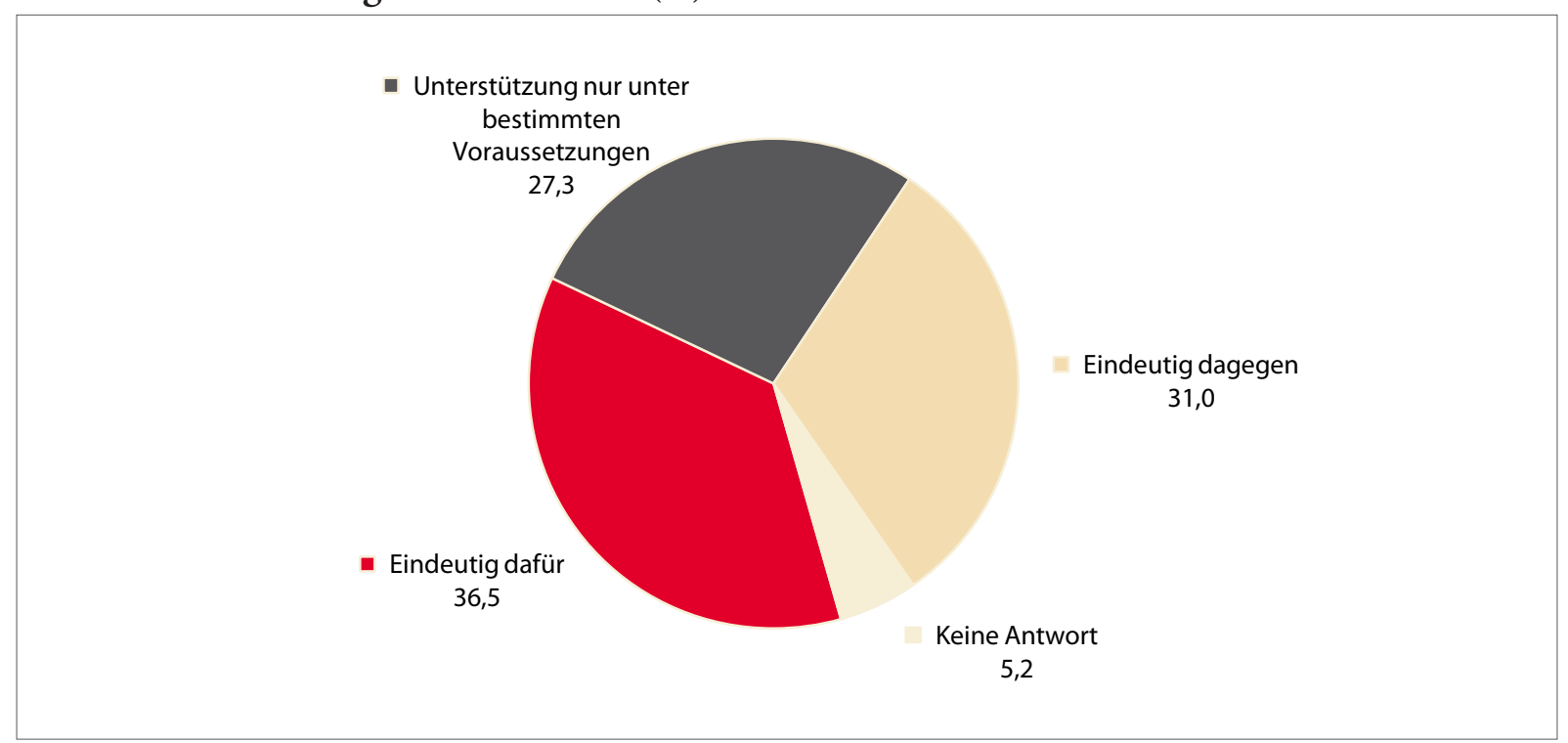

Quelle: A. Akulenko, O. Fablinowa, M. Tschernjanskaja: Prestuplenie i Nakasanie: Wosprijatie, ozenki, otnoschenie obschtschestwa [Verbrechen und Strafen: Wahrnehmung, Bewertungen, Einstellungen der Gesellschaft], Minsk 2013, <http://belhelcom.org/ru/node/18985>, S. 10

\section{Grafik 4: Einstellung zur Todesstrafe in Abhängigkeit vom Wohnort (\%)}

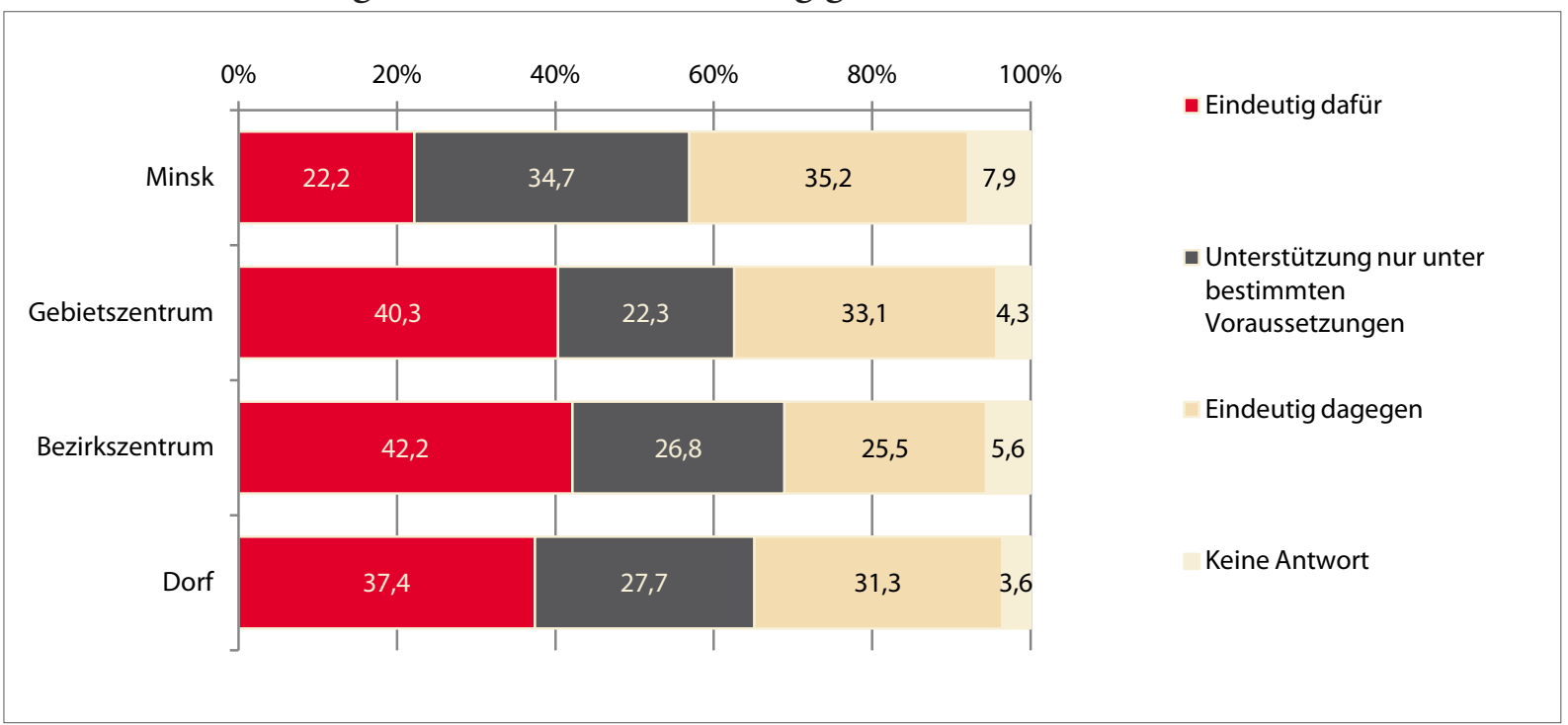

Quelle: A. Akulenko, O. Fablinowa, M. Tschernjanskaja: Prestuplenie i Nakasanie: Wosprijatie, ozenki, otnoschenie obschtschestwa [Verbrechen und Strafen: Wahrnehmung, Bewertungen, Einstellungen der Gesellschaft], Minsk 2013, <http://belhelcom.org/ru/node/18985>, S. 11 
Grafik 5: Einstellung zu Perspektiven der Anwendung der Todesstrafe (\%)

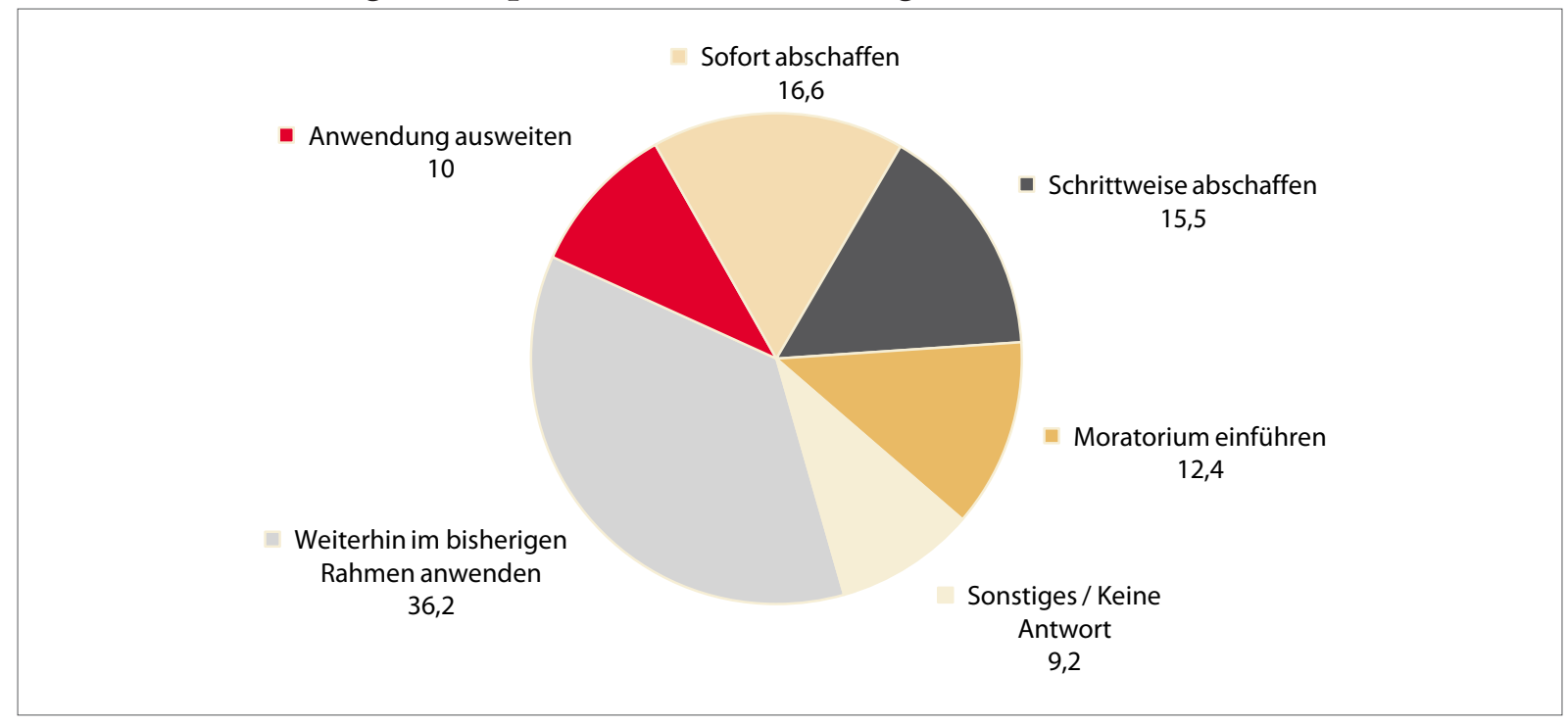

Quelle: A. Akulenko, O. Fablinowa, M. Tschernjanskaja: Prestuplenie i Nakasanie: Wosprijatie, ozenki, otnoschenie obschtschestwa [Verbrechen und Strafen: Wahrnehmung, Bewertungen, Einstellungen der Gesellschaft], Minsk 2013, <http://belhelcom.org/ru/node/18985>, S. 12

Grafik 6: Einstellung zu Perspektiven der Anwendung der Todesstrafe in Abhängigkeit vom Wohnort (\%)

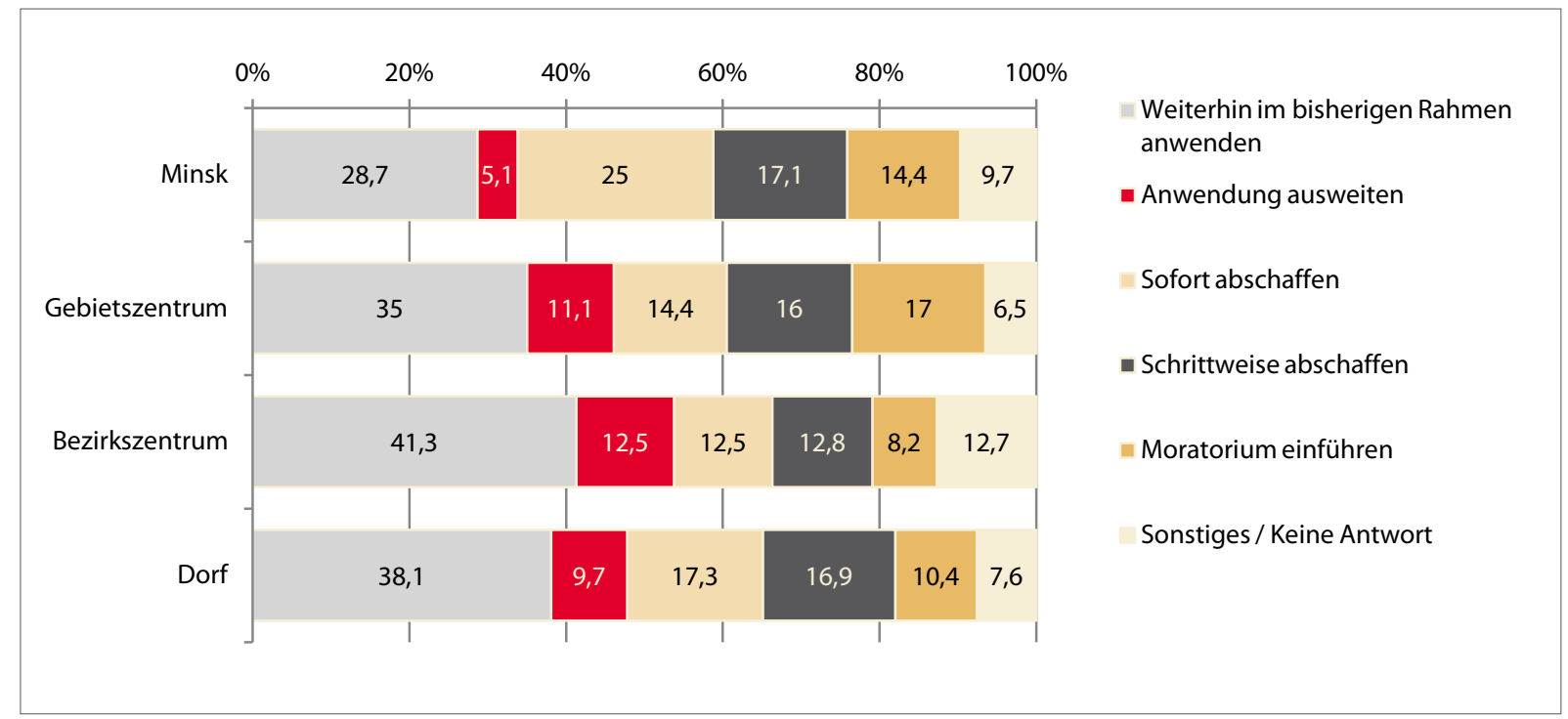

Quelle: A. Akulenko, O. Fablinowa, M. Tschernjanskaja: Prestuplenie i Nakasanie: Wosprijatie, ozenki, otnoschenie obschtschestwa [Verbrechen und Strafen: Wahrnehmung, Bewertungen, Einstellungen der Gesellschaft], Minsk 2013, <http://belhelcom.org/ru/node/18985>, S. 13 
Grafik 7: Einstellung zu Perspektiven der Anwendung der Todesstrafe in Abhängigkeit vom Glauben (\%)

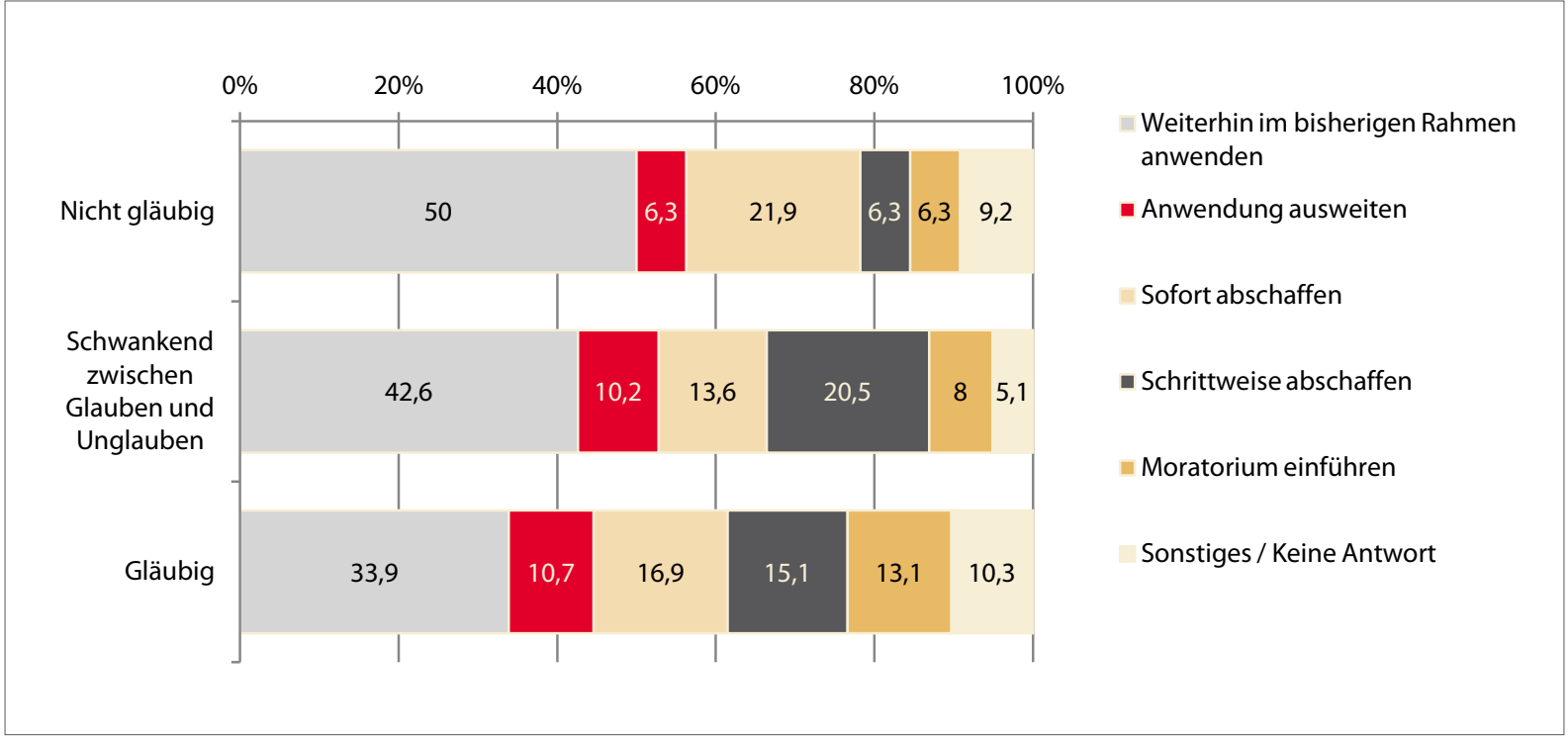

Quelle:A. Akulenko, O. Fablinowa, M. Tschernjanskaja:Prestuplenie i Nakasanie: Wosprijatie, ozenki, otnoschenie obschtschestwa [Verbrechen und Strafen: Wahrnehmung, Bewertungen, Einstellungen der Gesellschaft], Minsk 2013, <http://belhelcom.org/ru/node/18985>, S. 13

Grafik 8: Argumente für die Einführung eines Moratoriums (\%)

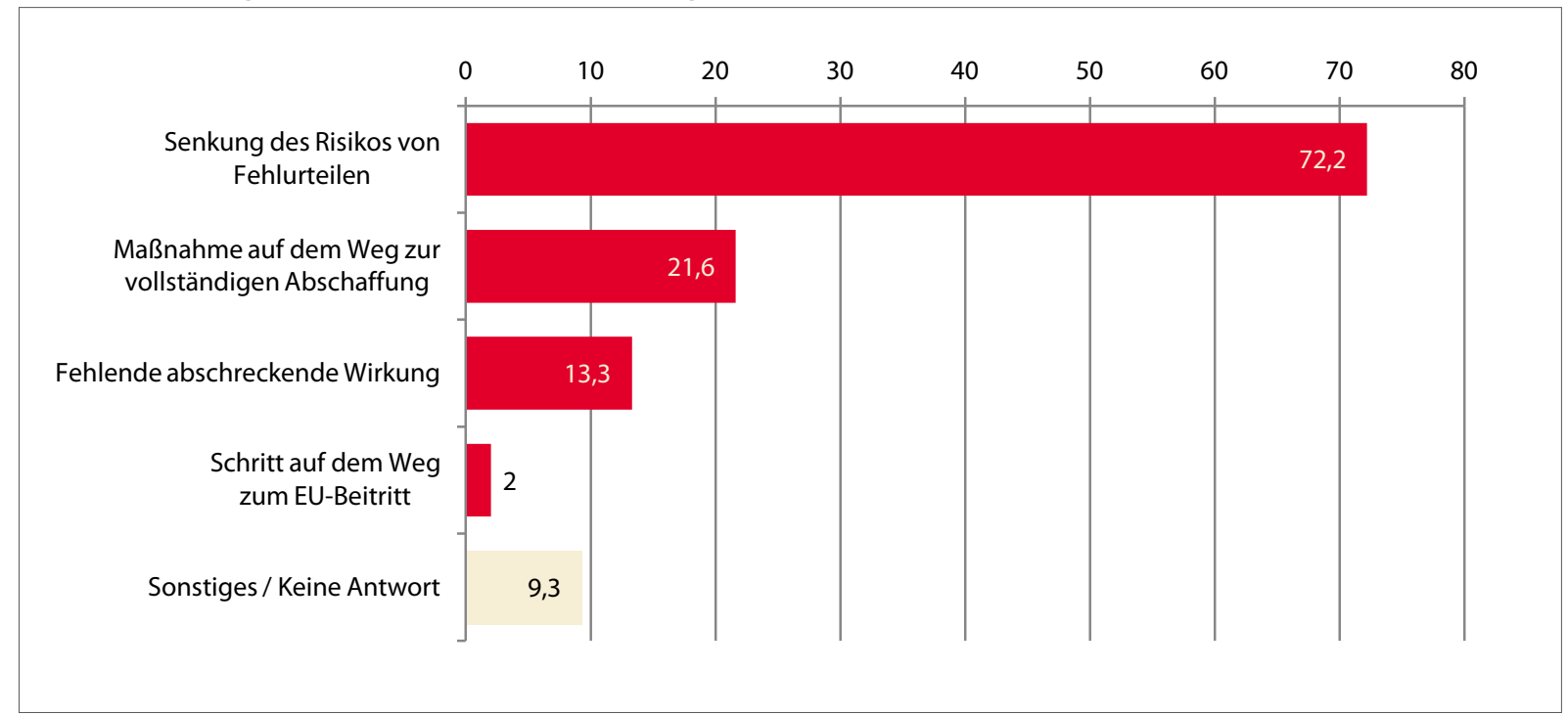

Quelle: A. Akulenko, O. Fablinowa, M. Tschernjanskaja: Prestuplenie i Nakasanie: Wosprijatie, ozenki, otnoschenie obschtschestwa [Verbrechen und Strafen: Wabrnehmung, Bewertungen, Einstellungen der Gesellschaft], Minsk 2013, <http://belhelcom.org/ru/node/18985>, S. 14 


\section{Die Unterstützung internationaler Geber für Belarus}

Grafik 1: Volumen der Fördermittel für Belarus aus EU und USA 2006-2011 (in Mio. US-Dollar)

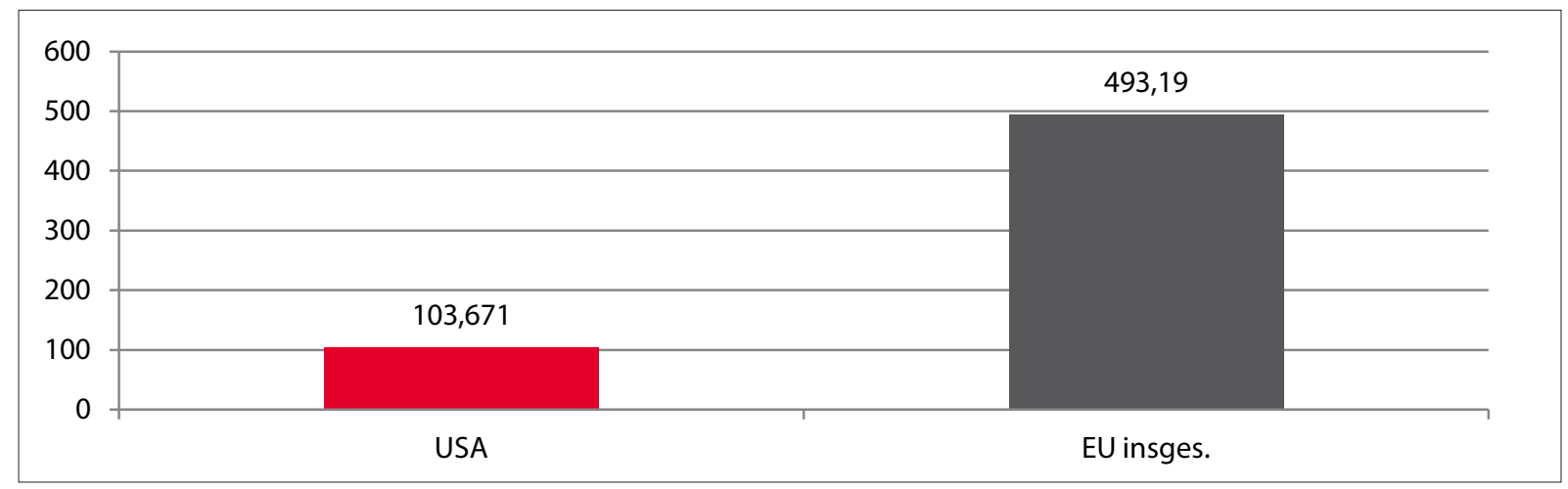

Grafik 2: Anteil der zentralen internationalen Geber an der internationalen Förderung für Belarus

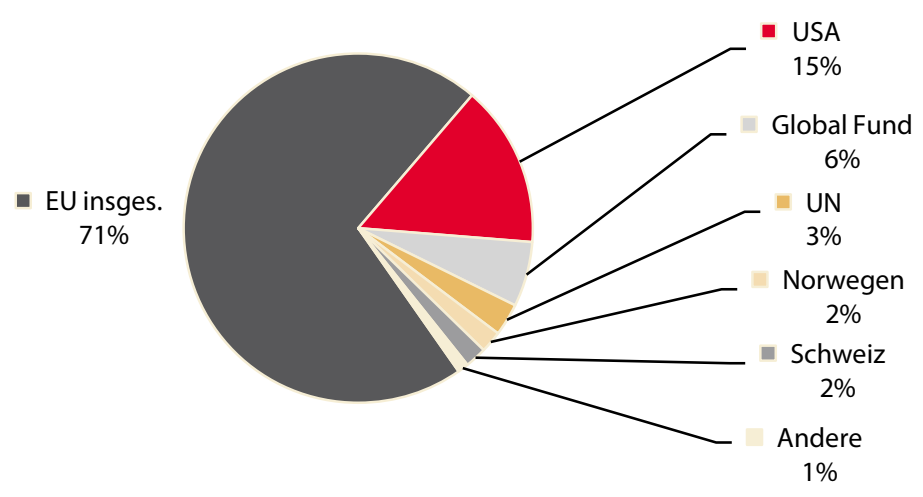

Grafik 3: Förderung durch EU und USA für Belarus im Bereich »Regierung und Zivilgesellschaft« (in Mio. US-Dollar)

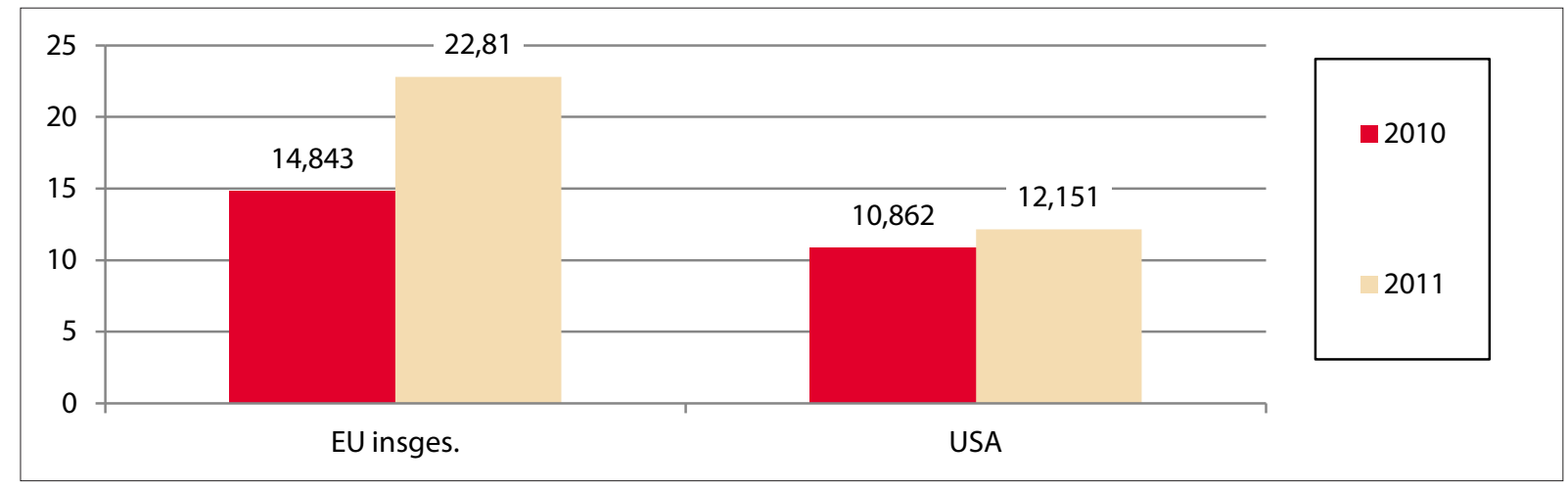

Quelle für alle Grafiken auf dieser Seite: Karyna Shyla / Andrei Yahorau: Civil Society's role and place in the system of the EU's donor assistance for Belarus, Working paper des Centre for European Transformation, Minsk 2013, http://cet.eurobelarus.info/en/news/2013/08/07/ civil-society-s-role-and-place-in-the-system-of-the-eu-s-donor-assistance-for-belarus-working-paper.html 


\section{Grafik 4: EU-Unterstützung für Belarus}

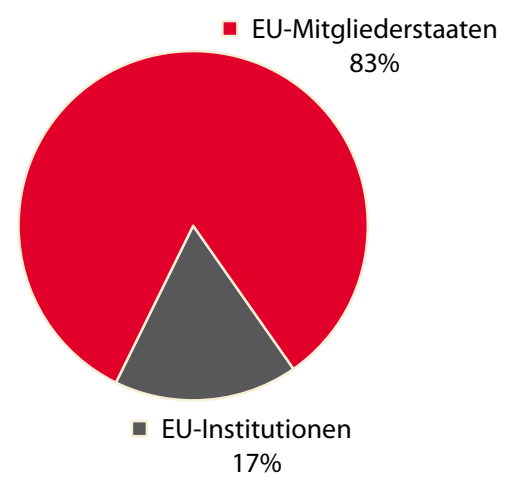

Grafik 5: Hilfe von EU-Mitgliedsstaaten 2006-2011

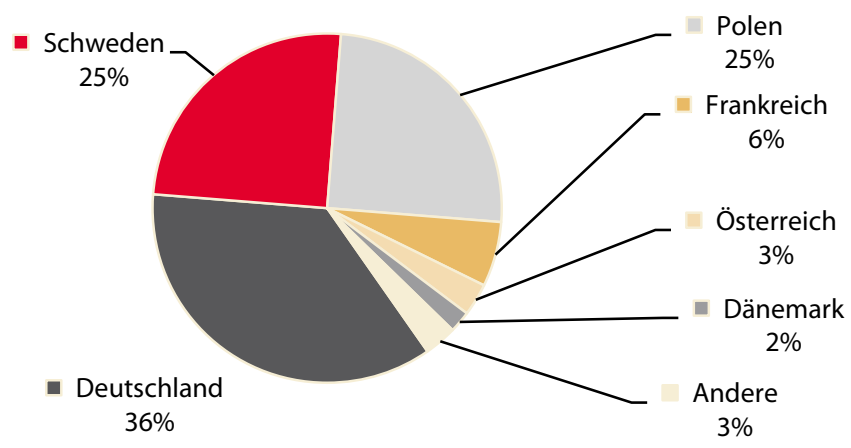

Grafik 6: Kanäle für die Bereitsstellung von EU-Hilfe für Belarus 2006-2011

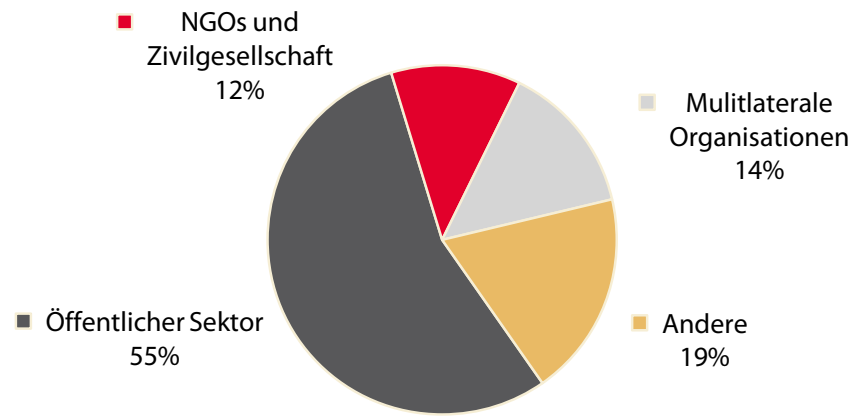

Quelle für alle Grafiken auf dieser Seite: Karyna Shyla / Andrei Yahorau: Civil Society's role and place in the system of the EU's donor assistance for Belarus, Working paper des Centre for European Transformation, Minsk 2013, http://cet.eurobelarus.info/en/news/2013/08/07/ civil-society-s-role-and-place-in-the-system-of-the-eu-s-donor-assistance-for-belarus-working-paper.html 


\section{Grafik 7: Hauptbereiche der EU-Förderung für Belarus}

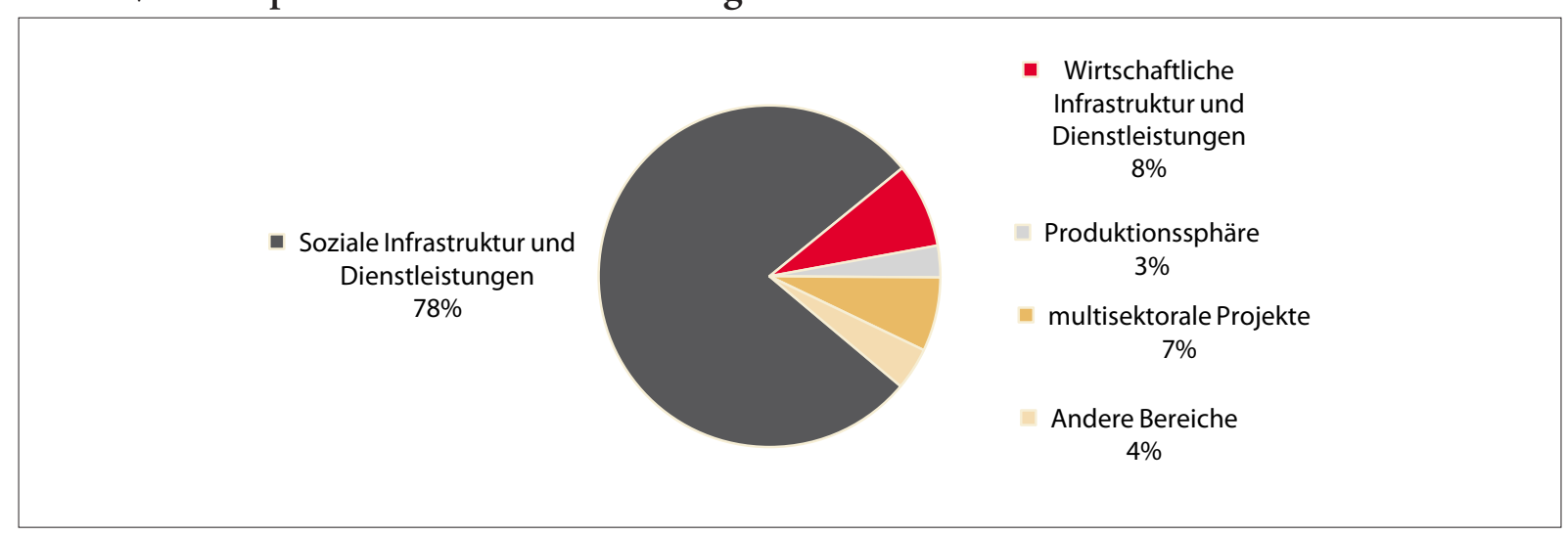

Grafik 8: Aufgliederung des Förderbereiches "Soziale Infrastruktur und Dienstleistungen« 2006-2011

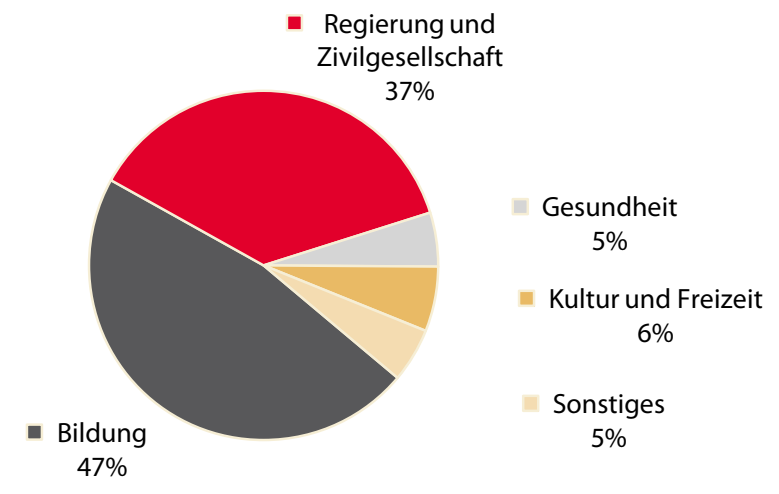

Quelle für alle Grafiken auf dieser Seite: Karyna Shyla / Andrei Yahorau: Civil Society's role and place in the system of the EU's donor assistance for Belarus, Working paper des Centre for European Transformation, Minsk 2013, http://cet.eurobelarus.info/en/news/2013/08/07/ civil-society-s-role-and-place-in-the-system-of-the-eu-s-donor-assistance-for-belarus-working-paper.html 


\section{Vom 16. Oktober bis 15. Dezember 2013}

\begin{tabular}{|c|c|}
\hline 17.10.2013 & $\begin{array}{l}\text { In Minsk findet die dritte Runde des technischen Dialogs zu wirtschaftlichen und finanzwirtschaftlichen Fra- } \\
\text { gen zwischen der Europäischen Kommission und der belarussischen Regierung statt. }\end{array}$ \\
\hline 18.10.2013 & $\begin{array}{l}\text { Belarussische Regierungsvertreter präsentieren Vertretern des Internationalen Währungsfonds und der Welt- } \\
\text { bank den Aktionsplan zur Durchführung von strukturellen Reformen zur Verbesserung der wirtschaftli- } \\
\text { chen Konkurrenzfähigkeit des Landes, den Regierung und Nationalbank am 10. Oktober beschlossen haben. }\end{array}$ \\
\hline 19.10.2013 & $\begin{array}{l}\text { Der stellvertretende Vorsitzende der Belarussischen Christdemokraten Pawal Sewjarynez wird nach dreijäh- } \\
\text { riger Haft entlassen, zu der er am 16. Mai } 2011 \text { wegen seiner Mitwirkung an den Protesten nach den Präsi- } \\
\text { dentschaftswahlen am 19. Dezember } 2010 \text { verurteilt worden war. Er hatte bereits von August } 2005 \text { bis Mai } \\
2007 \text { eine mehrjährige Haftstrafe wegen seiner Proteste gegen die Ergebnisse der Parlamentswahlen und des } \\
\text { Referendums in } 2004 \text { und zahlreiche kürzere Haftstrafen abgesessen. }\end{array}$ \\
\hline 19.10 .2013 & $\begin{array}{l}\text { Russland hebt das zeitweilige Einfuhrverbot für Schweinefleisch aus Belarus auf, das Ende August wegen der } \\
\text { Vorfälle von Schweinegrippe in Belarus verhängt worden war. }\end{array}$ \\
\hline 19.10.2013 & Die Belarussische Volksfront begeht den 25. Jahrestag ihrer Gründung. \\
\hline 20.10 .2013 & $\begin{array}{l}\text { Die Jugendorganisation der Belarussischen Christdemokraten hält in Minsk einen weiteren Gründungskon- } \\
\text { gress ab und wählt Maryna Chomitsch erneut zu ihrer Vorsitzenden. Der letzte Gründungskongress fand am } \\
\text { 3. November } 2012 \text { statt, doch das Justizministerium hatte im Februar } 2013 \text { die Registrierung verweigert. Am } \\
\text { 10. April } 2013 \text { hatte der Oberste Gerichtshof diese Entscheidung bestätigt. Insgesamt hat das Justizministe- } \\
\text { rium der Organisation seit } 2009 \text { bereits viermal die Registrierung verweigert. }\end{array}$ \\
\hline 21.10.2013 & $\begin{array}{l}\text { Präsident Lukaschenka warnt öffentlich vor einem zu schnellen Beitritt Armeniens zur Zollunion. Das Land } \\
\text { müsse erst alle Voraussetzungen erfüllen und seine Gesetzgebung anpassen, ehe es beitrete. Darüber hinaus } \\
\text { macht Lukaschenka deutlich, dass die Eurasische Wirtschaftskommission als supranationale Struktur zu ver- } \\
\text { stehen sei und daher alle Beteiligten einschließlich Russlands Souveränitätsrechte abtreten müssten. }\end{array}$ \\
\hline 21.10.2013 & $\begin{array}{l}\text { Ein Moskauer Gericht stellt einen Haftbefehl für den Generaldirektor von »Uralkali« Wladislaw Baumgertner } \\
\text { aus. Damit erfüllt Russland eine zentrale belarussische Forderung, um die Auslieferung des am 26. August } \\
2013 \text { in Minsk verhafteten Baumgertners zu erreichen. }\end{array}$ \\
\hline 22.10 .2013 & $\begin{array}{l}\text { Der Oberste Gerichtshof von Belarus hebt die Todesstrafe gegen Aljaksandr Hrunau auf und weist den Fall } \\
\text { an das zuständige Gericht für eine Neuverhandlung zurück. Der } 25 \text { Jahre alte Hrunau war am 14. Juni } 2013 \\
\text { wegen Mordes von einem Gomeler Gericht zum Tode verurteilt worden. Er hatte seine Schuld gestanden. }\end{array}$ \\
\hline 22.-23.10.2013 & $\begin{array}{l}\text { Der stellvertretende Außenminister Waljanzin Rybakau führt in Teheran Gespräche mit seinen iranischen } \\
\text { Amtskollegen, dem Minister für Industrie, Bergbau und Handel sowie mit Wirtschaftsvertretern. }\end{array}$ \\
\hline 22.-23.10.2013 & $\begin{array}{l}\text { Eine Delegation des OSZE-Büros für demokratische Institutionen und Menschenrechte (ODIHR) trifft sich } \\
\text { in Belarus mit Vertretern der Zentralen Wahlkommission sowie mit zivilgesellschaftlichen Akteuren und } \\
\text { Oppositionspolitikern. }\end{array}$ \\
\hline 24. -25.10 .2013 & $\begin{array}{l}\text { Die stellvertretende Außenministerin Aljona Kuptschyna empfängt in Minsk den politischen Direktor des } \\
\text { polnischen Außenministeriums Jaroslaw Bratkiewicz sowie den Generaldirektor für politische Angelegenhei- } \\
\text { ten des schwedischen Außenministeriums Torbjorn Sohlstrom. Gegenstand des Gesprächs, an dem auch der } \\
\text { Gehilfe des Präsidenten Valerij Bryljau teilnimmt, ist der bevorstehende EU-Gipfel in Vilnius. Die beiden } \\
\text { Diplomaten treffen sich außerdem mit Oppositionspolitikern, Journalisten und zivilgesellschaftlichen Akteuren. }\end{array}$ \\
\hline 25.10 .2013 & $\begin{array}{l}\text { In Minsk tagt der Oberste Eurasische Wirtschaftsrat unter Teilnahme der Präsidenten von Belarus, Kasachs- } \\
\text { tan und Russland. Um Proteste zu verhindern, hatten Sicherheitskräfte in den Tagen zuvor mehrere opposi- } \\
\text { tionelle Jugendaktivisten vorsorglich verhaftet oder unter Beobachtung gestellt. }\end{array}$ \\
\hline 25.10.2013 & $\begin{array}{l}\text { Mitarbeiter des belarussischen KGBs versuchen Medienberichten zufolge den stellvertretenden Direktor des } \\
\text { belarussischen Kalikartells Igor Jewstratow in Moskau zu verhaften. }\end{array}$ \\
\hline 26.-27.10.2013 & $\begin{array}{l}\text { An dem dritten Belarussischen Menschenrechtsforum in Vilnius nehmen } 110 \text { Vertreter von } 25 \text { belarussischen } \\
\text { Organisationen teil. Die Forumsteilnehmer sprechen sich in ihrer Erklärung u. a. für die Einrichtung einer } \\
\text { Vertretung des UN-Menschenrechtskommissars in sowie für die Erhaltung der Ad hoc Working Group der } \\
\text { Parlamentarischen Versammlung der OSZE zu Belarus aus. }\end{array}$ \\
\hline 27.10.2013 & $\begin{array}{l}\text { Beim Kongress der "Jungen Front« wird Smizer Daschkewitsch zu einem der } 4 \text { Ko-Vorsitzenden gewählt, } \\
\text { obwohl er nach seiner Freilassung aus dem Gefängnis Ende August } 2013 \text { zunächst seinen Austritt aus der } \\
\text { Organisation angekündigt hatte. }\end{array}$ \\
\hline
\end{tabular}




\begin{tabular}{|c|c|}
\hline 28.10 .2013 & $\begin{array}{l}\text { Die UN-Generalvollversammlung diskutiert in New York den von Miklos Haraszti erstellten Bericht über } \\
\text { die Menschenrechtssituation in Belarus. }\end{array}$ \\
\hline 29.10 .2013 & $\begin{array}{l}\text { Der Europäische Rat verlängert die gegen Belarus verhängten EU-Sanktionen um ein weiteres Jahr bis } 31 \text {. } \\
\text { Oktober 2014, da es weiterhin politische Gefangene im Land gebe und keine Fortschritte bei Menschenrech- } \\
\text { ten, Rechtsstaat und demokratischen Beziehungen erkennbar seien. Allerdings wird der Kreis der mit einem } \\
\text { Einreiseverbot belegten Belarussen verändert - Aufnahme von drei zusätzlichen Personen in die sowie Entfer- } \\
\text { nung von } 13 \text { Personen aus der Liste - so dass nun } 232 \text { Personen betroffen sind. Ebenso werden für } 5 \text { Firmen } \\
\text { die Sanktionen aufgehoben, für } 25 \text { weitere bleiben sie in Kraft. Das belarussische Außenministerium begrüßt } \\
\text { die Reduzierung der Sanktionen, bezeichnet ihre grundsätzliche Verlängerung jedoch als kontraproduktiv. } \\
\text { In den Reihen der Opposition gibt es unterschiedliche Wertungen: Während Wital Rymaschewskij von den } \\
\text { Belarussischen Christdemokraten die Kürzung der Sanktionsliste als Fehler kritisiert, wird dieser Schritt von } \\
\text { Aljaksandr Milinkewitsch (Bewegung für die Freiheit) ausdrücklich begrüßt. }\end{array}$ \\
\hline 29.10 .2013 & $\begin{array}{l}\text { Das litauische Außenministerium ruft die belarussische Führung auf, nicht mit dem AKW-Bau in Astrawez } \\
\text { zu beginnen, solange die Bewertung der Umweltauswirkungen des Projekts nicht entsprechend den Kriterien } \\
\text { der Espoo-Konvention abgeschlossen sei. }\end{array}$ \\
\hline 30.-31.10.2013 & $\begin{array}{l}\text { Der Präsident von Ecuador Rafael Vicente Correa Delgado hält sich zu seinem ersten Staatsbesuch in Belarus } \\
\text { auf. Im Gespräch mit Präsident Lukaschenka, der im Juli } 2012 \text { erstmals Ecuador besucht hatte, wird u. a. die } \\
\text { wechselseitige Eröffnung von Botschaften verabredet. Beide Seiten sprechen sich zudem für die Vertiefung } \\
\text { der wirtschaftlich-technischen Zusammenarbeit aus. }\end{array}$ \\
\hline $\begin{array}{l}31.10 .- \\
01.11 .2013\end{array}$ & $\begin{array}{l}\text { Außenminister Makej wird bei seinem Türkei-Besuch u. a. von Präsident Erdogan und seinem Amtskolle- } \\
\text { gen Ahmet Davutoglu empfangen. }\end{array}$ \\
\hline 01.11 .2013 & $\begin{array}{l}\text { Der Belarussische Kali-Konzern unterzeichnet ein Lieferabkommen mit dem chinesischen Kali-Importeuer } \\
\text { „Sinochem«. }\end{array}$ \\
\hline 02.11 .2013 & $\begin{array}{l}\text { Mit dem Präsidialerlass Nr. } 499 \text { genehmigt Aljaksandr Lukaschenka offiziell den Baubeginn des geplanten } \\
\text { Atomkraftwerks in Astrawez. }\end{array}$ \\
\hline 02.11 .2013 & $\begin{array}{l}\text { Präsident Lukaschenka entlässt den Chef des Grenzkomitees Aljaksandr Bajetschka und ernennt den ehema- } \\
\text { ligen Verteidigungsminister Leanid Malzau zu seinem Nachfolger. }\end{array}$ \\
\hline 02.11 .2013 & $\begin{array}{l}\text { Etwa } 400 \text { Personen nehmen an dem Gedenkmarsch zur Erinnerung an die Opfer des Stalinismus anlässlich } \\
\text { des Totengedenktags Dsjady nach Kurapaty teil. }\end{array}$ \\
\hline 04.11 .2013 & $\begin{array}{l}\text { Die Europäische Kommission legt ein neues, mit } 8 \text { Mio. Euro ausgestattetes Gesundheitsprogramm für die } \\
\text { Jahre 2014-2017 für Belarus auf mit dem Ziel, die Qualität der Gesundheitsfürsorge zu verbessern. }\end{array}$ \\
\hline 04.-05.11.2013 & $\begin{array}{l}\text { Präsident Lukaschenka trifft bei seinem zweitägigen Staatsbesuch in Turkmenistan u. a. mit seinem Amts- } \\
\text { kollegen Gurbanguly Berdimuhammedow zusammen. Ziel des Besuchs ist die Unterzeichnung mehrerer } \\
\text { Kooperationsabkommen, die insbesondere der Intensivierung der wirtschaftlichen Beziehungen dienen sol- } \\
\text { len. Gesprächsgegenstand ist außerdem die Umsetzung des Vertrags zum Aufbau eines Kalibergwerks nahe } \\
\text { dem Dorf Garlyk, zu dem sich Belarus im Januar } 2010 \text { vertraglich verpflichtet hatte. }\end{array}$ \\
\hline 05.11 .2013 & $\begin{array}{l}\text { Die russische Staatsanwaltschaft beantragt offiziell die Auslieferung des Generaldirektors von »Uralkali« Wla- } \\
\text { dislaw Baumgertner. }\end{array}$ \\
\hline 06.11 .2013 & $\begin{array}{l}\text { Der Ko-Vorsitzende der »Jungen Front«Smizer Daschkewitsch wird wegen des Vorwurfs der Missachtung von } \\
\text { Polizeianweisungen zu } 3 \text { Tagen Haft verurteilt. Daschkewitsch hatte in der Nähe des Kaufhauses „GUM» } \\
\text { Unterschriften zur Rückbenennung der Leninstraße in Franziskanerstraße gesammelt. }\end{array}$ \\
\hline 07.11 .2013 & $\begin{array}{l}\text { Präsident Lukaschenka hält anlässlich des Jahrestags der Oktober-Revolution eine Ansprache an die belarus- } \\
\text { sische Nation. Darin verweist er auf die Errungenschaften der Revolution und auf die gute Tradition, den } \\
\text { Arbeitern an diesem Tag Geschenke zu machen. Das diesjährige Geschenk bestehe in der Fertigstellung von } \\
\text { neuem Wohnraum und neuen Produktionsanlagen, worin sich die Verbesserung des Lebensstandards der } \\
\text { Menschen ausdrücke. Belarus ist das einzige postsowjetische Land, in dem der Jahrestag der Oktoberevolu- } \\
\text { tion nach wie vor ein gesetzlicher Feiertag ist. }\end{array}$ \\
\hline 08.11 .2013 & $\begin{array}{l}\text { Präsident Lukaschenka besucht den holzverarbeitenden Betrieb»Barysaudreu«, um die Umsetzung des Moder- } \\
\text { nisierungsprogramms zu überprüfen. Noch während des Besuchs enthebt Lukaschenka aufgrund seiner } \\
\text { Unzufriedenheit mit dem vorgefundenen Zustand des Betriebs den stellvertretenden Leiter der Präsidialad- } \\
\text { ministration Andrej Tur, den Gouverneur des Minsker Gebiets Barys Batura und den Leiter des Konzerns } \\
\text { "Bellespaperrapram« Aljaksandr Peraslawzau ihrer Ämter. }\end{array}$ \\
\hline
\end{tabular}




\begin{tabular}{|c|c|}
\hline 9.-16.11.2013 & $\begin{array}{l}\text { Eine belarussische Delegation unter Leitung des stellvertretenden Außenministers Aljaksandr Hurjanau besucht } \\
\text { Brasilien. Beide Seiten unterzeichnen ein Abkommen über Visafreiheit für Kurzreisen. }\end{array}$ \\
\hline 12.11.2013 & $\begin{array}{l}\text { Bei seinem Inspektionsbesuch in der Fliesenfabrik »Keramin« kündigt Präsident Lukaschenka an, dass er } \\
\text { allen großen Betrieben des Landes einen Kontrollbesuch abstatten werde, um die Ausführung seiner Anord- } \\
\text { nungen zu überprüfen. }\end{array}$ \\
\hline 14.11 .2013 & $\begin{array}{l}\text { Aljaksandr Lukaschenka ernennt den bisherigen Gouverneur des Gebiets Hrodna Sjamjon Schapira zum } \\
\text { Nachfolger von Barys Batyra als Vorsitzenden des Minsker Gebietsexekutivkomitees. Neuer Hrodnaer Gou- } \\
\text { verneur wird der Parlamentsabgeordnete Uladzimir Krawzau, der bis } 2012 \text { stellvertretender Leiter des Mahil- } \\
\text { jauer Gebietsexekutivkomitees war. }\end{array}$ \\
\hline 18.11 .2013 & $\begin{array}{l}\text { Das Oberste Wirtschaftsgericht weist die Klage des Verlegers Ihar Lohwinau gegen den Entzug der Lizenz sei- } \\
\text { nes Verlages durch das Informationsministerium im September } 2013 \text { zurück. Als Anlass für den Lizenzentzug } \\
\text { diente die Herausgabe des Bandes der belarussischen Pressefotos des Jahres 2011, das als »extremistisch» ein- } \\
\text { gestuft wurde. Lohwinau kündigt an Berufung einzulegen und nach weiteren Möglichkeiten zu suchen, um } \\
\text { die Tätigkeit seines in } 2000 \text { gegründeten Verlags im In- oder Ausland fortsetzen zu können. }\end{array}$ \\
\hline 19.11 .2013 & $\begin{array}{l}\text { Angela Merkel spricht im Deutschen Bundestag zum EU-Gipfel in Vilnius und betont, dass eine engere } \\
\text { Kooperation mit Belarus erst nach der Freilassung und Rehabilitation aller politischen Gefangenen möglich } \\
\text { sei. Sie äußert die Hoffnung, dass die belarussische Delegation den Gipfel nutzen werde, um ein Zeichen für } \\
\text { ihre diesbezügliche Bereitschaft zu setzen. }\end{array}$ \\
\hline 19.11 .2013 & $\begin{array}{l}\text { Aljaksandr Lukaschenka unterzeichnet ein Gesetz, das die Umwandlung von gesellschaftlichen Vereinigungen } \\
\text { in politische Parteien erlaubt, wenn sie mindestens } 1000 \text { Mitglieder in zahlreichen Regionen des Landes hat. }\end{array}$ \\
\hline 19.11.2013 & $\begin{array}{l}\text { Außenminister Uladsimir Makej erklärt nach einem Gespräch mit seinem russischen Amtskollegen Lawrow, } \\
\text { dass Belarus keine besonderen Erwartungen in Verbindung mit dem EU-Gipfel in Vilnius hege und Belarus des- } \\
\text { wegen nicht auf hohem Niveau beim Gipfel vertreten sein werde. Allerdings sei Belarus an einer Einbeziehung } \\
\text { in die "Östliche Partnerschaft« interessiert, da dies einer Normalisierung der Beziehungen zur EU dienlich sei. }\end{array}$ \\
\hline 20.-21.11.2013 & $\begin{array}{l}\text { Präsident Lukaschenka trifft während seines Staatsbesuchs in Aserbaidschan mit seinem Amtskollegen Ilham } \\
\text { Alijew zusammen. Beide Präsidenten weihen das neue Gebäude der belarussischen Botschaft in Baku ein } \\
\text { und betrachten Fahrzeuge, die eine lokale Firma unter Einsatz von Fertigungsteilen der Minsker Automo- } \\
\text { bilwerke und der Minsker Traktorenwerke produziert. Es werden außerdem mehrere Kooperationsabkom- } \\
\text { men unterzeichnet. }\end{array}$ \\
\hline 21.11 .2013 & $\begin{array}{l}\text { Der seit 26. August } 2013 \text { in Minsk inhaftierte Generaldirektor von „Uralkali« Wladislaw Baumgertner wird } \\
\text { zwecks Auslieferung nach Russland in die russische Botschaft in Minsk gebracht und anschließend nach Mos- } \\
\text { kau ausgeflogen, wo er entsprechend den bilateralen Absprachen in ein russisches Gefängnis gebracht wird. }\end{array}$ \\
\hline 22.11 .2013 & $\begin{array}{l}\text { Die stellvertretende Außenministerin Aljona Kuptschyna empfängt in Minsk eine Delegation des litauischen } \\
\text { Außenministeriums unter Leitung des stellvertretenden litauischen Außenministers Andrius Krivas, um die } \\
\text { bilateralen Beziehungen sowie die Beziehungen zwischen Belarus und der EU im Kontext des Vilnius-Gip- } \\
\text { fels zu diskutieren. }\end{array}$ \\
\hline 25.11 .2013 & $\begin{array}{l}\text { Dem Staatlichen Presseamt zufolge wird Außenminister Makej für Belarus an dem EU-Gipfel zur »Östlichen } \\
\text { Partnerschaft« in Vilnius teilnehmen. Wirtschaftsminister Snapkau wird das Land beim Business-Forum ver- } \\
\text { treten, das einen Tag vor dem Gipfel ebenfalls in Vilnius stattfindet. }\end{array}$ \\
\hline 27.-30.11.2013 & $\begin{array}{l}\text { Eine Delegation unter Leitung des stellvertretenden Außenministers Aljaksandr Hurjanau nimmt in Frank- } \\
\text { reich an der Generalversammlung des International Exhibitions Bureau (BIE) teil. Die Delegation führt } \\
\text { außerdem Gespräche im französischen Außenministerium und im Außenhandelsministerium sowie im } \\
\text { OECD-Hauptquartier. }\end{array}$ \\
\hline 28.11 .2013 & $\begin{array}{l}\text { Im litauischen Parlament findet eine Anhörung zu Belarus statt, zu der zahlreiche Oppositionspolitiker und } \\
\text { Menschenrechtler eingeladen sind. }\end{array}$ \\
\hline 28.11 .2013 & $\begin{array}{l}\text { Wirtschaftsminister Snapkau erklärt beim Business-Forum in Vilnius, dass Belarus seine aktuellen Liqui- } \\
\text { ditätsprobleme durch Eurobonds sowie Kredite des Internationalen Währungsfonds und russischer Banken } \\
\text { zu lösen hoffe. Er betont gleichzeitig die Bereitschaft zu Reformen, um die Wettbewerbsfähigeit staatlicher } \\
\text { Betriebe zu erhöhen. Gleichzeitig betont er, dass Belarus zu Recht bisher auf eine umfassende Privatisierung } \\
\text { im Unterschied zu seinen Nachbarstaaten verzichtet habe. }\end{array}$ \\
\hline 28.11 .2013 & $\begin{array}{l}\text { Außenminister Makej nimmt am informellen Abendessen zum Auftakt des Dritten EU-Gipfels zur "Östli- } \\
\text { chen Partnerschaft« in Vilnius teil. Belarus ist das einzige Teilnehmerland der "Östlichen Partnerschaft«, das } \\
\text { nicht durch seinen Präsidenten oder Regierungschef vertreten ist. }\end{array}$ \\
\hline
\end{tabular}




\begin{tabular}{|c|c|}
\hline 28.-29.11.2013 & $\begin{array}{l}\text { Außenminister Makej erklärt beim EU-Gipfel in Vilnius, die Bereitschaft seines Landes, Verhandlungen } \\
\text { über ein Visaerleichterungsabkommen mit der EU zu beginnen. Er betont, es handele sich dabei um einen } \\
\text { Vorschlag von Präsident Lukaschenka im Interesse der eigenen Bevölkerung, und verweist auf frühere ent- } \\
\text { sprechende belarussische Initiativen aus dem Jahre } 2004 \text {. Makej erklärt außerdem, dass er die Erklärung des } \\
\text { Vilnius-Gipfels grundsätzlich unterstütze, obwohl er mit einigen Punkten nicht einverstanden sei. Die Dekla- } \\
\text { ration betont die wichtige Rolle von Belarus für die europäische Energiesicherheit sowie die bilaterale Koope- } \\
\text { ration von Belarus und EU in konkreten Bereichen und begrüßt die Beteiligung von Belarus am EU-Stress- } \\
\text { test für im Betrieb oder in Planung befindliche Atomreaktoren. Gleichzeitig erklärt die EU ihre Bereitschaft, } \\
\text { den Modernisierungsdialog mit Belarus fortzusetzen. }\end{array}$ \\
\hline 29.11.2013 & $\begin{array}{l}\text { Der Ko-Vorsitzende der Christdemokraten Pawal Sewjarynez, der Vorsitzende der vereinigten Bürgerpartei } \\
\text { Anatol Ljabedska und die Ehefrau des inhaftierten Menschenrechtlers Natallja Pintschuk nehmen am Rande } \\
\text { des EU-Gipfels in Vilnius an einem Treffen mit der litauischen Präsidentin Dalia Grybauskaite teil. }\end{array}$ \\
\hline 29.11.2013 & $\begin{array}{l}\text { Aljaksandr Lukaschenka droht die sofortige Entlassung des Premierministers und seiner Stellvertreter an für } \\
\text { den Fall, dass die Wirtschaftsziele für das erste Quartal } 2014 \text { nicht erreicht werden. }\end{array}$ \\
\hline 01.-05.12.2013 & $\begin{array}{l}\text { Bauminister Anatol Tschorny und der erste stellvertretende Landwirtschaftsminister Leanid Marynitsch besu- } \\
\text { chen Venezuela, um den Fortgang bilateraler Projekte zu begutachten. }\end{array}$ \\
\hline 02.12 .2013 & $\begin{array}{l}\text { Aljaksandr Lukaschenka kündigt an, dass die Vorsitzende der Zentralen Wahlkommission Lidsija Jarmoschyna } \\
\text { ihr Amt auch bei den in den nächsten Jahren anstehenden Wahlen ausüben soll. In } 2014 \text { stehen Lokalwah- } \\
\text { len an, in } 2015 \text { Präsidentschaftswahlen. }\end{array}$ \\
\hline 02.12 .2013 & $\begin{array}{l}\text { Die russische Regierung erhöht die für Dezember in Aussicht gestellte Öllieferung von } 300.000 \text { auf } 750.000 \\
\mathrm{~m}^{3} \text {. Belarus wird damit in } 2013 \text { voraussichtlich 19,25 Mio. t anstelle der erwarteten } 23 \text { Mio. t Öl aus Russ- } \\
\text { land erhalten. }\end{array}$ \\
\hline 2.-6.12. 2013 & $\begin{array}{l}\text { Mitglieder der Belarus-Parlamentariergruppe des französischen Parlaments führen in Belarus Gespräche mit } \\
\text { Außenminister Makej, dem Vorsitzenden des Rats der Republik Rubinau sowie Vertretern des Repräsentan- } \\
\text { tenhauses und des Wirtschaftsministeriums. Sie besuchen außerdem die Belarussischen Automobilwerke in } \\
\text { Shodino, das Französisch-Belarussische Zentrum für Europäische Studien sowie die Minsker Universität für } \\
\text { Linguistik. }\end{array}$ \\
\hline 03.12 .2013 & $\begin{array}{l}\text { Der im Juni } 2013 \text { verhaftete katholische Priester Uladsislau Lasar wird aus dem KGB-Gefängnis in Minsk } \\
\text { entlassen. Die Untersuchung gegen ihn wird jedoch weiter geführt. Dem Priester wird die Beteiligung an } \\
\text { Spionage im Interesse eines fremden Staates vorgeworfen. }\end{array}$ \\
\hline 04.12 .2013 & $\begin{array}{l}\text { Der Generaldirektor des russischen Düngerproduzenten »Uralchim», der einen 20prozentigen Anteil an »Ural- } \\
\text { kali« erwirbt, erklärt, dass seine Firma keine Vorbehalte gegen eine Wiederherstellung des gemeinsamen Kar- } \\
\text { tells mit "Belaruskali« habe. Gleichzeitig erklärt er, dass das Unternehmen bisher auch nicht die Absetzung } \\
\text { von Wladislaw Baumgertner als Generaldirektor von »Uralkali« verfolge. }\end{array}$ \\
\hline 5.-6.12.2013 & Außenminister Makej nimmt am 20. Treffen des OSZE-Ministerrats in Kiew teil. \\
\hline 09.12 .2013 & $\begin{array}{l}\text { Aktivisten der "Jungen Front« eröffnen ein belarussisches Stabsbüro im Kiewer Rathaus, das von den Pro- } \\
\text { testteilnehmern gegen die Politik von Präsident Janukowitsch besetzt wurde. Zu den belarussischen Protest- } \\
\text { teilnehmern gehören u. a. der Vorsitzende der Belarussischen Volksfront Aljaksej Janukewitsch und der stell- } \\
\text { vertretende Vorsitzende der Bewegung für die Freiheit Juryj Hubarewitsch, die am 5. Dezember auf dem } \\
\text { Unabhängigkeitsplatz sprechen durften, sowie der Vorsitzende der Bewegung »Sag die Wahrheit!» Uladsimir } \\
\text { Njakljajeu, der am 6. Dezember eine Redemöglichkeit erhielt. }\end{array}$ \\
\hline 10.12 .2013 & $\begin{array}{l}\text { Metropolit Filaret schreibt in einem Brief an die Mutter des wegen zweifachen Mordes zum Tode verurteilten } \\
\text { Pawel Seljun, dass die orthodoxe Kirche sich für die Abschaffung der Todesstrafe einsetze. Der Brief wurde } \\
\text { außerdem beim Begnadigungsausschuss der Präsidialadministration eingereicht. Während seiner bisherigen } \\
\text { Präsidentschaft hat Aljaksandr Lukashenka bisher allerdings nur einmal, im Jahre 2003, einem Gnadenge- } \\
\text { such stattgegeben und die Todesstrafe in eine 15jährige Haftstrafe umgewandelt. }\end{array}$ \\
\hline 10.12 .2013 & $\begin{array}{l}\text { Der in einem Moskauer Gefängnis sitzende Generaldirektor von »Uralkali« Wladislaw Baumgertner wird in } \\
\text { Hausarrest überführt. Das Untersuchungsverfahren gegen ihn läuft weiter. }\end{array}$ \\
\hline 10.12 .2013 & $\begin{array}{l}\text { Das Minsker Stadtexekutivkomitee verbietet eine Demonstration im Rahmen der Kampagne Gay Pride } 2013 . \\
\text { Die diesjährige Kampagne steht unter dem Motto „Wir existieren« und soll im Umfeld des Weltmenschen- } \\
\text { rechtstags die öffentliche Aufmerksamkeit auf die Probleme homosexueller Menschen in Belarus lenken. }\end{array}$ \\
\hline
\end{tabular}




\begin{tabular}{|l|l|}
\hline 10.-11.12.2013 & $\begin{array}{l}\text { Der US-Unterstaatssekretär für Europa und Eurasien Eric Rubin trifft in Minsk mit Außenminister Makej, } \\
\text { Oppositionspolitikern und zivilgesellschaftlichen Akteuren zusammen. Die USA erwarten von Minsk die } \\
\text { Aufhebung der Begrenzung der Zahl ihrer Botschaftsmitarbeiter. Im Falle einer Verbesserung der Menschen- } \\
\text { rechts- und Demokratielage in Belarus könnten die USA außerdem Rubin zufolge leichter einem IWF-Kre- } \\
\text { dit für Belarus zustimmen, da die Rechtslage des Landes die Berücksichtigung dieser Kriterien bei der Ent- } \\
\text { scheidung verlange. Rubin betont gleichzeitig, dass die USA nur über weniger als 20\% der Stimmen im IWF } \\
\text { verfüge und diese Entscheidung daher nicht alleine zu treffen habe. }\end{array}$ \\
\hline 12.12.2013 & $\begin{array}{l}\text { Das Repräsentantenhaus bestätigt den Staatshaushalt für } 2014 \text { in erster Lesung. Der Haushalt geht von einem } \\
\text { BIP-Wachstum von 3,3\% aus. Die Einführung einer neuen Steuer für KFZ-Besitzer wird ebenfalls in erster } \\
\text { Lesung angenommen. Die Idee, eine Steuer für nicht-arbeitende Personen im erwerbsfähigen Alter einzufüh- } \\
\text { ren, scheint die Regierung hingegen aufgegeben zu haben. }\end{array}$ \\
\hline 12.-13.12.2013 & $\begin{array}{l}\text { Der stellvertretende Außenminister Aljaksandr Hurjanau hält sich zu Gesprächen zur Vorbereitung der Sit- } \\
\text { zung der belarussisch-polnischen Kommission zur grenzüberschreitenden Zusammenarbeit in Polen auf. }\end{array}$ \\
\hline 13.12.2013 & $\begin{array}{l}\text { Das Industrieministerium bestätigt Berichte über Lohnkürzungen in den Minsker Traktorenwerken aufgrund } \\
\text { von Absatzschwierigkeiten. In den Werken standen zum 1. November 9.900 Traktoren auf Lager. Dies ent- } \\
\text { spricht 179\% der monatlichen Produktion. Im November war die Produktion bereits um 8,1\% auf 5.500 } \\
\text { Traktoren gedrosselt worden. }\end{array}$ \\
\hline
\end{tabular}

Zusammengestellt auf der Grundlage der Meldungen der Nachrichten-Agentur BelaPAN und der Homepage naviny.by.

Sie können die gesamte Chronik ab dem 14.03.2011 auch auf<http://www.laender-analysen.de/belarus/> unter dem Link »Chronik«lesen.

Die Belarus-Analysen werden von der Alfried Krupp von Bohlen und Halbach-Stiftung unterstützt.

\section{Alfried Krupp von Bohlen und Halbach Stiftung}

Herausgeber: Forschungsstelle Osteuropa an der Universität Bremen, Deutsche Gesellschaft für Osteuropakunde e.V. und Internationales Bildungs- und Begegnungswerk, Dortmund

Die Meinungen, die in den Belarus-Analysen geäußert werden, geben ausschließlich die Auffassung der Autoren wieder. Abdruck und sonstige publizistische Nutzung sind nach Rücksprache mit der Redaktion gestattet.

Redaktion: Astrid Sahm (verantwortlich) Satz: Matthias Neumann

Belarus-Analysen-Layout: Cengiz Kibaroglu, Matthias Neumann und Michael Clemens Alle Ausgaben der Belarus-Analysen sind mit Themen- und Autorenindex archiviert unter www.laender-analysen.de ISSN 2192-1350 @ 2013 by Forschungsstelle Osteuropa, Bremen 


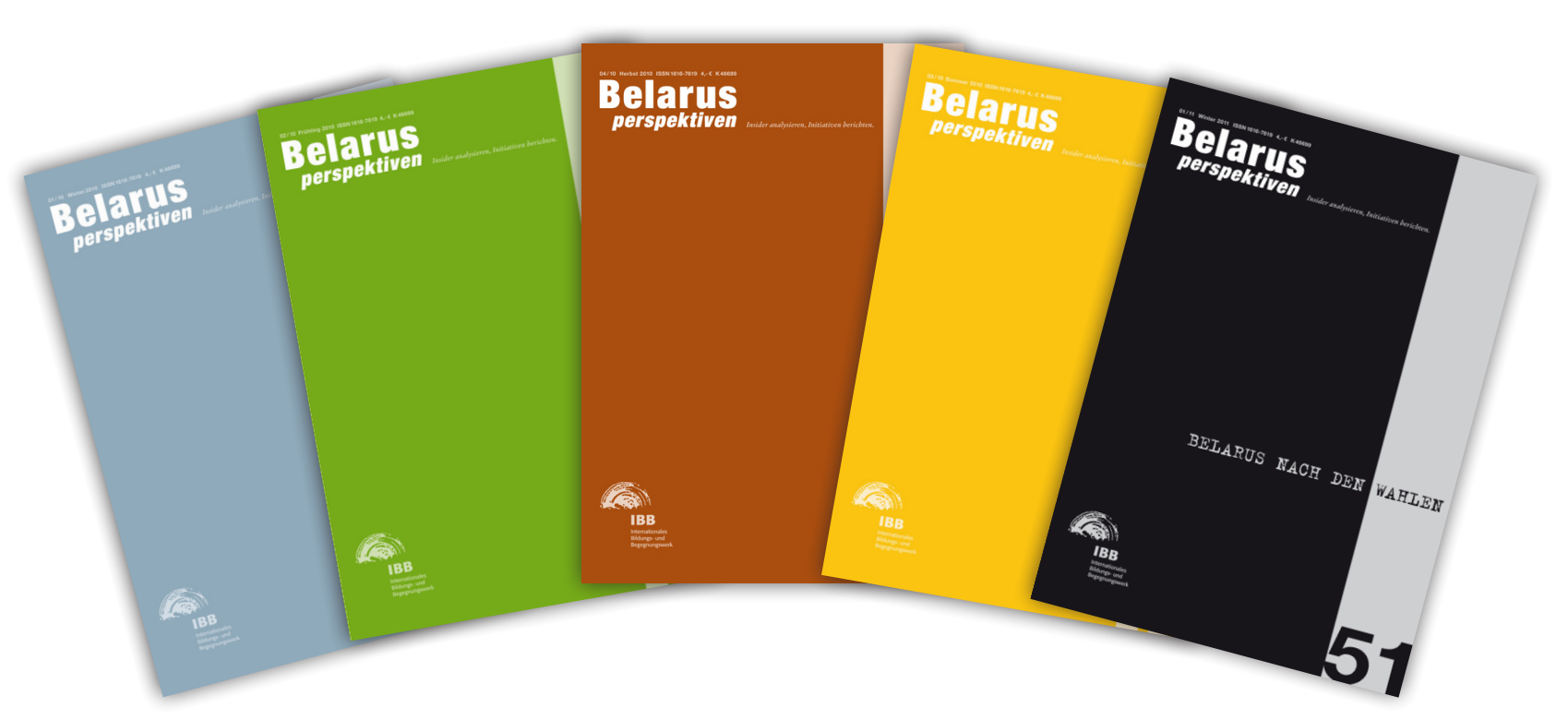

\section{Belarus \\ Perspektiven}

- Seit mehr als zehn Jahren berichten die Belarus Perspektiven aus einem fast unbekannten Land. Uns lesen Initiativen und Politiker, Journalisten und Unternehmer - all jene, die in Belarus etwas bewegen wollen.

- Durch unsere kritischen Berichte und Analysen sind unsere Leser nicht nur auf dem neuesten Stand sie erfahren auch, was hinter den Kulissen geschieht.

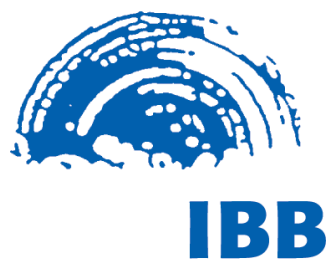

Internationales Bildungs- und Begegnungswerk
- Autoren der Belarus Perspektiven sind namhafte belarussische Journalisten, unabhängige Medienvertreter, Wirtschaftsexperten und zivilgesellschaftliche Akteure.

- Die Zeitschrift erscheint vierteljährlich und kostet inklusive Versand $15,-€$ im Jahresabonnement.

- Zu beziehen sind die Belarus Perspektiven beim IBB in Dortmund bzw. online unter <http://www.ibb-d.de/publikation-anfordernhtml.html>

- Ältere Ausgaben der Belarus Perspektiven finden Sie im Archiv als pdf-Datei zum Download, <http://www.ibb-d.de/bp-archiv.html> 


\section{Kostenlose E-Mail-Dienste der Forschungsstelle Osteuropa und ihrer Partner auf www.laender-analysen.de}

Die Länder-Analysen bieten regelmäßig kompetente Einschätzungen aktueller politischer, wirtschaftlicher, sozialer und kultureller Entwicklungen in Ostmitteleuropa und der GUS. Sie machen das Wissen, über das die wissenschaftliche Forschung in reichem Maße verfügt, für Politik, Wirtschaft, Medien und die interessierte Öffentlichkeit verfügbar. Autoren sind internationale Fachwissenschaftler und Experten.

Die einzelnen Länder-Analysen werden von der Forschungsstelle Osteuropa an der Universität Bremen und der Deutschen Gesellschaft für Osteuropakunde jeweils mit unterschiedlichen Partnern und Sponsoren herausgegeben.

Die Länder-Analysen bieten regelmäßig Kurzanalysen zu aktuellen Themen, ergänzt um Grafiken und Tabellen sowie Dokumentationen. Zusätzlich gibt es eine Chronik aktueller Ereignisse.

\section{Belarus-Analysen \\ Erscheinungsweise: zweimonatlich \\ Abonnement unter: fsopr@uni-bremen.de}

\section{Caucasus Analytical Digest}

In englischer Sprache. Erscheinungsweise: monatlich

Abonnement unter: <http://www.res.ethz.ch/analysis/cad/>

\section{Polen-Analysen}

Erscheinungsweise: zweimal monatlich

Abonnement unter: <http://www.deutsches-polen-institut.de/Newsletter/subscribe.php>

\section{Russland-Analysen}

Erscheinungsweise: zweiwöchentlich

Abonnement unter: fsopr@uni-bremen.de

\section{Russian Analytical Digest}

In englischer Sprache. Erscheinungsweise: zweimal monatlich

Abonnement unter: <http://www.res.ethz.ch/analysis/rad/>

\section{Ukraine-Analysen}

Erscheinungsweise: zweimal monatlich

Abonnement unter: fsopr@uni-bremen.de

\section{Zentralasien-Analysen}

Erscheinungsweise: monatlich

Abonnement unter: zentralasien-analysen@dgo-online.org

\section{Bibliographische Dienste}

Die Bibliographien informieren über englisch- und deutschsprachige Neuerscheinungen zu Belarus, Polen, Russland, Tschechischer und Slowakischer Republik, Ukraine sowie zu den zentralasiatischen und kaukasischen Staaten. Erfasst werden jeweils die Themenbereiche Politik, Außenpolitik, Wirtschaft und Soziales.

Erscheinungsweise: viermal jährlich

Abonnement unter: fsopr@uni-bremen.de 